Sharif University of Technology
Scientia Iranica
Transactions E: Industrial Engineering
hCIENTIA

\title{
On the Bayesian analysis of two-component mixture of transmuted Weibull distribution
}

\author{
R. Yousaf ${ }^{a}$, S. Ali ${ }^{\text {b,*, }}$, and M. Aslam ${ }^{a}$ \\ a. Department of Mathematics and Statistics, Riphah International University, Islamabad, Pakistan. \\ b. Department of Statistics, Quaid-i-Azam University, Islamabad, 45320, Pakistan.
}

Received 23 May 2018; received in revised form 18 December 2018; accepted 2 September 2019

\author{
KEYWORDS \\ Transmuted Weibull \\ distribution; \\ Mixture model; \\ Loss functions; \\ Bayes estimators; \\ Posterior risks; \\ Uniform prior; \\ Informative prior; \\ Bayesian intervals; \\ MCMC; \\ Type-I right \\ censoring.
}

\begin{abstract}
Transmuted distributions are skewed distributions that have recently attracted the attention of researchers due to their applications in reliability and statistics. In this article, the main focus is on the Bayesian estimation of a two-component mixture of the Transmuted Weibull Distribution (TWD) under a type-I right censored sampling scheme. In order to estimate the unknown parameters, non-informative and informative priors under Squared Error Loss Function (SELF), Precautionary Loss Function (PLF) and Quadratic Loss Function (QLF) are assumed when computing the posterior estimations. In addition, the Bayesian Credible Intervals (BCI) are also constructed. A Markov Chain Monte Carlo (MCMC) technique is adopted to generate samples from the posterior distributions and, in turn, to compute different posterior summaries, including Bayes Estimates (BEs), Posterior Risks (PRs) and BCI. As an illustration, comparison of these Bayes estimators is made through simulation under different loss functions in terms of their respective PRs, assuming different sample sizes and censoring rates. Two real-life examples, the first being the survival times of hepatitis B \& C patients, while the second being a hole diameter of $12 \mathrm{~mm}$ and a sheet thickness of $3.15 \mathrm{~mm}$, are also discussed to illustrate the potential application of the proposed methodology.
\end{abstract}

(C) 2021 Sharif University of Technology. All rights reserved.

\section{Introduction}

Shaw and Buckley [1] introduced a technique to construct skewed distributions by using the Quadratic Rank Transmutation Map (QRTM) to generate a flexible family of probability distributions. The authors considered the extreme value distribution as the base line distribution and used a transmuted parameter to enhance their flexibility [2]. Currently, transmuted distributions are applied in many diverse fields such

*. Corresponding author. Tel.: 00925190642185 E-mail addresses: ejazraheela@yahoo.com (R. Yousaf); sajidali.qau@hotmail.com (S. Ali); m.aslam@riphah.edu.pk (M. Aslam)

doi: $10.24200 /$ sci. 2019.51090 .1997 as reliability studies, lifetime analysis, engineering, economics, medicine, insurance and environmental sciences [3].

A random variable $X$ is said to follow a transmuted distribution if its Probability Density Function (PDF) and Cumulative Distribution Function (CDF) can be written as:

$$
\begin{aligned}
& f(x)=g(x)\{1+\lambda-2 \lambda G(x)\}, \\
& F(x)=(1+\lambda) G(x)-\lambda G^{2}(x),
\end{aligned}
$$

where $x>0$ and $|\lambda| \leq 1$ is the transmuted parameter, and $g(x)$ and $G(x)$ are the PDF and CDF of the baseline distribution, respectively.

The Weibull distribution is a popular continuous probability distribution introduced by Swedish physicist Waloddi Weibull in 1939 in order to describe the 
behavior of the breaking strength of materials [4]. Since its inception, the Weibull distribution has proved to be a versatile lifetime distribution and many other distributions are its special cases based on the different values of the shape parameter. The Weibull distribution has been extensively used for analyzing lifetime data in reliability engineering, astronomy, medicine, psychology, botany, zoology, agriculture, fisheries, aerospace, and electronics [5]. However, to generalize the Weibull distribution for a non-monotonic hazard rate, several distributions have been introduced in the literature. The first generalization of the Weibull distribution with application to survival data is given by Mudholkar et al. [6]. Later, Aryal and Tsokos [7] introduced the Transmuted Weibull Distribution (TWD) which is a generalization of Weibull probability distribution. Khan and King [8] introduced the transmuted modified Weibull distribution which is more flexible than the TWD. The new modified distribution reduces to the TWD by setting the scale parameter equal to zero. Merovci et al. [9] proposed the transmuted generalized inverse Weibull distribution and discussed some of its mathematical properties. The authors also estimated the parameters using the method of maximum likelihood. Khan et al. [5] introduced transmuted generalized Weibull distribution using the QRTM technique and explored its mathematical properties including the expressions for the quantile function, moments, entropies, mean deviation, Bonferroni and Lorenz curves and moments of the order statistics. The authors also estimated the model parameters using the method of maximum likelihood. This distribution is an important competitive model that contains twenty three lifetime distributions as special cases. More recently, Abdurrahman [10] used the method of maximum likelihood and the method of moment estimators to estimate its parameters. Similarly, Nofal and El Gebaly [11] proposed a generalized TWD based on a generalized transmuted-G family and derived its properties. The main feature of this distribution is that two additional parameters are inducted in the $\mathrm{PDF}$ and $\mathrm{CDF}$ of Weibull distribution to provide greater flexibility for the generated distribution and contains eleven lifetime distributions as special cases. The generalized TWD can be reduced into a Transmuted Class (TC) studied by Shaw and Buckley [1], by equating two additional parameters equal to one.

Finite mixture models provide a flexible framework to handle heterogeneous data with a finite number of unobserved subpopulations and also have been widely applied to classification, clustering, and pattern identification problems. The mixture models have received a great deal of attention in the recent era due to their flexibility. A finite mixture of probability distributions is suitable to study a population categorized in a number of subpopulations mixed in an unknown proportion. The concept of the finite mixture distribution was pioneered by Newcomb [12] for modeling outliers. The mixture models can be used even when available data are generated from a mixture of two or more distributions. This was the authors' motivation behind mixing two or more statistical models to get a new mixture model. The analysis of mixture models under the Bayesian framework has gained significant interest among statisticians. Most researchers have worked on two-component mixture models using both classical and Bayesian analysis. Feroze and Aslam [13] presented the Bayesian analysis of doubly censored lifetime data using a two-component mixture of the Weibull distribution. Sindhu et al. [14] studied the two-component mixture of inverse Weibull distributions under a doubly censored sample using various loss functions. Aslam et al. [15,16] and Tahir et al. [17] studied the properties of a three-component mixture of Rayleigh distributions, while Ateya [18] discussed the mixture of generalized exponential distribution. Similarly, Benaicha and Chaker [19] also studied the mixture of the Weibull distribution. These contributions to the mixture models are great motivators for recent studies.

Motivated by the popularity of the mixture models, this study considers the Bayesian parameter estimation of a two-component mixture of the TWD. Bayesian estimation is done assuming different noninformative (uniform) and informative (gamma and inverse gamma) priors, and three loss functions are used to obtain the posterior summaries. Censoring is an important aspect of lifetime data because most times it is not possible to continue the experiment until the last observation, in order to obtain a complete data set. A censored data set contains at least one observation about which only partial information on the exact failure time is available. There are three types of censored observation, the left, the interval and the right censored observations. Due to the nonavailability of the true lifetime of certain objects, type-I right censoring is used by taking some pre-specified test termination time [20-22], e.g., if a patient survives until the end of a study, the patient's time of death is rightcensored. Since the marginal posterior distributions are not in closed forms, we proposed a new Markov Chain Monte Carlo (MCMC) algorithm in order to obtain Bayes Estimates (BEs), Posterior Risks (PRs) and 95\% credible intervals.

The remainder of the article is structured as follows: The transmuted Weibull mixture model, sampling scheme, likelihood function, expressions of posterior distributions using noninformative and informative priors, and marginal posterior densities for censored data are discussed in Section 2. The expressions for the Bayes Estimators (BEs) and their respective PRs under different loss functions are presented in Section 
3. To obtain the posterior summaries, an MCMC algorithm is presented in Section 3. The results of BEs and their PRs based on a simulation study are tabulated in Section 4. In Section 5, the Bayesian Credible Intervals (BCI) are discussed mathematically and numerically. Two real-life data sets are presented in Section 6, while some concluding remarks are given in Section 7.

\section{The mixture of transmuted Weibull model}

In this section, we introduce the likelihood function for a two-component mixture of TWD.

A random variable $X$ is said to follow a finite mixture distribution with m-components and unknown mixing proportion $(p)$, if the PDF of $X$ can be written as:

$$
f(x \mid \theta)=\sum_{i=1}^{m} p_{i} f\left(x \mid \theta_{i}\right)
$$

where $p_{i}>0 ; i=1,2, \ldots, m$ and $\sum_{i=1}^{m} p_{i}=1$.

The parameter $p_{i}$ is the mixing proportion of the $i$ th component, whereas $f_{i}(x)$ denotes the density of the $i$ th component parameterized by $\theta_{i}$.

A finite two-component mixture model of TWD with unknown mixing proportions $p_{1}$ and $p_{2}$ has the following form:

$$
\begin{aligned}
& f(x ; \Omega)=p_{1} f_{1}\left(x ; \Omega_{1}\right)+\left(1-p_{1}\right) f_{2}\left(x ; \Omega_{2}\right) \\
& p_{2} \leq 1-p_{1},
\end{aligned}
$$

where $\Omega=\left(\alpha_{1}, \alpha_{2}, \beta_{1}, \beta_{2}, \lambda_{1}, \lambda_{2}, p_{1}\right), \Omega_{i}=\left(\alpha_{i}, \beta_{i}, \lambda_{i}\right)$, $i=1,2$, and $f_{i}\left(x ; \Omega_{i}\right)$ denote the PDF of the $i$ th component that can be written as:

$$
\begin{aligned}
f_{i}\left(x ; \Omega_{i}\right) & =p_{i} \frac{\alpha_{i}}{\beta_{i}} x_{i}^{\alpha_{i}-1} \exp \left(-\frac{x_{i}{ }^{\alpha_{i}}}{\beta_{i}}\right) \\
& \left\{1-\lambda_{i}+2 \lambda_{i} \exp \left(-\frac{x_{i}{ }^{\alpha_{i}}}{\beta_{i}}\right)\right\},
\end{aligned}
$$

$\mathrm{x}_{\mathrm{i}} \geq 0, \alpha_{i}, \beta_{i}>0$, and $\left|\lambda_{i}\right| \leq 1, \quad i=1,2$.

Further, the CDF of a two-component mixture of TWD is:

$$
F(x ; \Omega)=p_{1} F_{1}\left(x ; \Omega_{1}\right)+\left(1-p_{1}\right) F_{2}\left(x ; \Omega_{2}\right),
$$

where the CDF of the $i$ th component is given by:

$$
\begin{aligned}
F_{i}\left(x ; \Omega_{i}\right) & =p_{i} \exp \left(-\frac{x^{\alpha_{i}}}{\beta_{i}}\right) \\
& \left\{1-\lambda_{i}+\lambda_{i} \exp \left(-\frac{x^{\alpha_{i}}}{\beta_{i}}\right)\right\}
\end{aligned}
$$

$\mathrm{x}_{\mathrm{i}} \geq 0, \quad \alpha_{i}, \beta_{i}>0, \quad\left|\lambda_{i}\right| \leq 1$ and $i=1,2$.

\section{Special cases}

- If $\lambda=0$ and $p_{1}=1$ then the two-component mixture of the TWD is reduced to the Weibull distribution;

- If $\alpha=1$ and $p_{1}=1$ then transmuted exponential distribution occurs. In addition, the exponential distribution is obtained by assuming $\lambda=0$;

- If $\alpha=\beta=1$ and $p_{1}=1$, then the resulting distribution is the transmuted standard exponential distribution;

- If $\alpha=2$ and $p_{1}=1$, then the transmuted Rayleigh distribution is obtained. Further, if $\lambda=0$, then the ordinary Rayleigh distribution is obtained.

\subsection{Sampling scheme}

Suppose that $n$ units are put on a life testing experiment with a fixed termination time $t$. At the end of the experiment, it is found that $r$ units out of $n$ objects have failed and $n-r$ objects are still functioning. As noted by Mendenhall and Hader [23], in many real-life situations, only the failed units can easily be categorized into either a member of subpopulation-I or subpopulation-II. For example, an engineer can identify whether a failed electronic object is a member of the first or the second subpopulation based on the cause of its failure. Thus, out of $r$ failures, $r_{1}$ and $r_{2}$ failures belong to subpopulation-I and subpopulation-II, respectively. However, $r=r_{1}+r_{2}$ denotes the number of observed observations while $n-r$ observations are censored. Now, $x_{l j}, 0<x_{l j}<t$ is defined as the failure time of the $j$ th object belonging to the $l$ th subpopulation, where $l=1,2$ and $j=1,2, \ldots, r_{l}$.

\subsection{The likelihood function}

For a 2-component mixture model, the likelihood function is:

$$
\begin{aligned}
L(\mathbf{x} ; \Omega)= & \left\{\prod_{j=1}^{r_{1}} p_{1} f_{1}\left(x_{1 j}\right)\right\}\left\{\prod_{j=1}^{r_{2}} p_{2} f_{2}\left(x_{2 j}\right)\right\} \\
& \{1-F(T)\}^{n-r},
\end{aligned}
$$

where:

$$
\begin{aligned}
& \Omega=\left(\alpha_{1}, \alpha_{2}, \beta_{1}, \beta_{2}, \lambda_{1}, \lambda_{2}, p_{1},\right. \\
& \mathbf{x}=\left(x_{11}, x_{12}, \ldots x_{1 r_{1}}, x_{21}, x_{22}, \ldots x_{2 r_{2}}\right),
\end{aligned}
$$

and $F(T)$ denote the CDF at time $(T)$.

For the TWD, the likelihood expression can be written as:

$$
\begin{aligned}
L(x ; \Omega)= & {\left[\prod_{j=1}^{r_{1}} p_{1} \frac{\alpha_{1}}{\beta_{1}} x_{1 j}^{\alpha_{1}-1} \exp \left(-\frac{x_{1 j}{ }^{\alpha_{1}}}{\beta_{1}}\right)\right.} \\
& \left.\left\{1-\lambda_{1}+2 \lambda_{1} \exp \left(-\frac{x_{1 j}^{\alpha_{1}}}{\beta_{1}}\right)\right\}\right]
\end{aligned}
$$




$$
\begin{aligned}
& {\left[\prod_{j=1}^{r_{21}}\left(1-p_{1}\right) \frac{\alpha_{2}}{\beta_{2}} x_{2 j}^{\alpha_{2}-1} \exp \left(-\frac{x_{2 j}{ }^{\alpha_{2}}}{\beta_{2}}\right)\right.} \\
& \left.\left\{1-\lambda_{2}+2 \lambda_{2} \exp \left(-\frac{x_{2 j}^{\alpha_{2}}}{\beta_{2}}\right)\right\}\right] \\
& {\left[1-p_{1} \exp \left(-\frac{T^{\alpha_{1}}}{\beta_{1}}\right)\right.} \\
& \left\{1-\lambda_{1}+2 \lambda_{1} \exp \left(-\frac{T^{\alpha_{1}}}{\beta_{1}}\right)\right\} \\
& -\left(1-p_{1}\right) \exp \left(-\frac{T^{\alpha_{2}}}{\beta_{2}}\right) \\
& \left.\left\{1-\lambda_{2-}+2 \lambda_{2} \exp \left(-\frac{T^{\alpha_{2}}}{\beta_{2}}\right)\right\}\right]^{n-r},
\end{aligned}
$$

which can be simplified as:

$$
\begin{gathered}
L(x ; \Omega) \propto p_{1}^{E_{11}-1}\left(1-p_{1}\right)^{E_{12}-1} \alpha_{1}^{E_{11}-1} \exp \left(-\alpha_{1} F_{11}\right) \\
\alpha_{2}^{E_{12}-1} \exp \left(-\alpha_{2} F_{12}\right) \frac{1}{\beta_{1}^{E_{21}+1}} \exp \left(-\frac{G_{11}}{\beta_{1}}\right) \\
\frac{1}{\beta_{2}^{E_{22}+1}} \exp \left(-\frac{G_{12}}{\beta_{2}}\right) \exp \left(H_{11}\right) \exp \left(H_{12}\right) I^{n-r},
\end{gathered}
$$

where:

$$
\begin{aligned}
& E_{11}=r_{1}+1, \quad E_{12}=r_{2}+1, \\
& F_{11}=\sum_{j=1}^{r_{1}} \log \left(\frac{1}{x_{1 j}}\right), \quad F_{12}=\sum_{j=1}^{r_{2}} \log \left(\frac{1}{x_{2 j}}\right), \\
& E_{21}=r_{1}-1, \quad E_{22}=r_{2}-1, \\
& G_{11}=\sum_{j=1}^{r_{1}} x_{1 j}^{\alpha_{2}}, \quad G_{12}=\sum_{j=1}^{r_{1}} x_{2 j}^{\alpha_{2}}, \\
& H_{11}=\sum_{j=i}^{r_{1}} \log \left\{1-\lambda_{1}+2 \lambda_{1} \exp \left(-\frac{x_{1 j}^{\alpha_{1}}}{\beta_{1}}\right)\right\} \\
& H_{12}=\exp \left[\sum_{j=i}^{r_{2}} \log \left\{1-\lambda_{2}+2 \lambda_{2} \exp \left(-\frac{x_{2 j}^{\alpha_{2}}}{\theta_{2}}\right)\right\}\right] \\
& I=1-p_{1} \exp \left(-\frac{T^{\alpha_{1}}}{\beta_{1}}\right)\left\{1-\lambda_{1}+2 \lambda_{1} \exp \left(-\frac{T^{\alpha_{1}}}{\beta_{1}}\right)\right\} \\
& -\left(1-p_{1}\right) \exp \left(-\frac{T^{\alpha_{2}}}{\beta_{2}}\right)\left\{1-\lambda_{2}+\lambda_{2} \exp \left(-\frac{T^{\alpha_{2}}}{\beta_{2}}\right)\right\} .
\end{aligned}
$$

In the next section, posterior distribution is discussed assuming different priors.

\subsection{The posterior distribution using Uniform Prior (UP)}

The main difference between the Bayesian and classical inference is prior information. The prior distribution uses past information about a phenomenon under investigation. Selecting an appropriate prior is definitely the most important task of the Bayesian analysis [24,25], because posterior distribution depends heavily on prior information. There are situations where sufficient prior information regarding the parameter of interest is available. An important noninformative prior, proposed by Laplace [26], is the uniform prior, which has been applied to many problems, and mostly provides satisfactory results. The noninformative uniform prior for $\alpha_{1}, \alpha_{2}, \beta_{1}, \beta_{2}, \lambda_{1}, \lambda_{2}$ and $p_{1}$ is defined as:

$$
\begin{array}{ll}
\alpha_{1} \sim \operatorname{Uniform}(0, \infty) & \alpha_{2} \sim \operatorname{Uniform}(0, \infty) \\
\beta_{1} \sim \operatorname{Uniform}(0, \infty) & \beta_{2} \sim \operatorname{Uniform}(0, \infty) .
\end{array}
$$

Furthermore, the UP over the interval $(0,1)$ is assumed for mixing proportion $p_{1}$, i.e., $p_{1} \sim \operatorname{Uniform}(0,1)$, while the priors for transmuted parameters $\lambda_{1}$ and $\lambda_{2}$ are $U P(-1,1)$. In this study, it is assumed that the prior distributions of $\alpha_{1}, \alpha_{2}, \beta_{1}, \beta_{2}, \lambda_{1}, \lambda_{2}$ and $p_{1}$ are independent $[27,28]$. The joint prior distribution of parameters $\alpha_{1}, \alpha_{2}, \beta_{1}, \beta_{2}, \lambda_{1}, \lambda_{2}$ and $p_{1}$ is:

$$
\begin{aligned}
& \pi_{U P}(\Omega) \propto 1 ; \quad \alpha_{1}, \alpha_{2}>0, \quad \beta_{1}, \beta_{2}>0, \\
& -1<\lambda_{1}, \quad \lambda_{2}<1, \quad 0<p_{1}<1,
\end{aligned}
$$

By the Bayes theorem:

$$
g(\Omega \mid x)=\frac{L(x ; \Omega) \pi(\Omega)}{\int_{\Omega}^{\infty} L(x ; \Omega) \pi(\Omega d \Omega)},
$$

where $\pi(\Omega)$ denotes the joint prior distribution of:

$$
\begin{aligned}
& \Omega=\left(\alpha_{1}, \alpha_{2}, \beta_{1}, \beta_{2}, \lambda_{1}, \lambda_{2}, p_{1}\right), \\
& \mathbf{x}=\left(x_{11}, x_{12}, \ldots x_{1 r_{1}}, x_{21}, x_{22}, \ldots x_{2 r_{2}}\right) .
\end{aligned}
$$

$L(x ; \Omega)$ denotes the likelihood function and $g(\Omega \mid x)$ is the joint posterior distribution; the joint posterior distribution is calculated by Eq. (6) as shown in Box I.

The marginal posterior density of each parameter can be obtained by integrating the joint posterior distribution with respect to the nuisance parameter. For example:

$$
\begin{gathered}
g_{U P}\left(\alpha_{1} \mid x\right)=\int_{0}^{\infty} \int_{0}^{\infty} \int_{0}^{\infty} \int_{-1}^{1} \int_{-1}^{1} \int_{0}^{1} g_{U P}(\Omega \mid x) \\
d p_{1} d \lambda_{1} d \lambda_{2} d \beta_{1} d \beta_{2} d \alpha_{2} .
\end{gathered}
$$


$g_{U P}(\Omega \mid x)=$

$$
\begin{aligned}
& p_{1}^{E_{11}}\left(1-p_{1}\right)^{E_{12}-1} \alpha_{1}^{E_{11}-1} \exp \left(-\alpha_{1} F_{11}\right) \alpha_{2}^{E_{12}-1} \exp \left(-\alpha_{2} F_{12}\right) \frac{1}{\beta_{1}^{E_{21}+1}} \exp \left(-\frac{G_{11}}{\beta_{1}}\right) \frac{1}{\beta_{2}^{E} 2^{+1}} \\
& \exp \left(-\beta_{2} G_{12}\right) \exp \left(H_{11}\right) \exp \left(H_{12}\right)(K)^{n-r} \\
& \int_{0}^{\infty} \int_{0}^{\infty} \int_{0}^{\infty} \int_{0}^{\infty} \int_{-1}^{1} p_{1}^{E_{11}}\left(1-p_{1}\right)^{E_{12}-1} \alpha_{1}^{E_{11}-1} \exp \left(-\alpha_{1} F_{11}\right) \alpha_{2}^{E_{12}-1} \exp \left(-\alpha_{2} F_{12}\right) \frac{1}{\beta_{1}^{E}{ }_{21+1}} \exp \left(-\frac{G_{11}}{\beta_{1}}\right) \frac{1}{\beta_{2}^{E_{22}+1}} \exp \left(-\beta_{2} G_{12}\right)
\end{aligned}
$$

Box I

\subsection{The posterior distribution using Informative Prior (IP)}

If some specific and definite information about the values of the parameters is available, it is quantified as an informative prior and must be included in the analysis. For the mixture of TWD, different prior distributions are assumed, such as gamma, inverse gamma, uniform, and beta for $\alpha_{1}, \alpha_{2}, \beta_{1}, \beta_{2}, \lambda_{1}, \lambda_{2}$ and $p_{1}$. To this end, let $\alpha_{1} \sim \operatorname{gamma}\left(a_{1}, b_{1}\right), \alpha_{2} \sim$ $\operatorname{gamma}\left(a_{2}, b_{2}\right), \beta_{1} \sim$ inverse $-\operatorname{gamma}\left(c_{1}, d_{1}\right), \beta_{2} \sim$ inverse $-\operatorname{gamma}\left(c_{2}, d_{2}\right) \lambda_{1} \sim \operatorname{Uniform}\left(l_{1}, l_{2}\right), \lambda_{2} \sim$ Uniform $\left(m_{1}, m_{2}\right)$ and $p_{1} \sim \operatorname{beta}\left(e_{1}, e_{2}\right)$. These priors are selected by keeping in mind the range of the parameters [29]. Assuming independence, one has joint prior distribution of the parameters $\alpha_{1}, \alpha_{2}, \beta_{1}, \beta_{2}, \lambda_{1}, \lambda_{2}$, and $p_{1}$ :

$$
\begin{gathered}
\pi_{I P}(\Omega) \propto \alpha_{1}^{a_{1}-1} e^{-b_{1} \alpha_{1}} \alpha_{2}^{a_{2}-1} e^{-b_{2} \alpha_{2}} \frac{1}{\beta_{1}^{c_{1}+1}} e^{-\frac{d_{1}}{\beta_{1}}} \\
\frac{1}{\beta_{2}^{c_{2}+1}} e^{-\frac{d_{2}}{\beta_{2}}} p_{1}^{e_{1}-1}\left(1-p_{1}\right)^{e_{2}-1} .
\end{gathered}
$$

The joint posterior distribution of parameters $\alpha_{1}, \alpha_{2}$, $\beta_{1}, \beta_{2}, \lambda_{1}, \lambda_{2}$, and $p_{1}$ given data $x$ is calculated by Eq. (7) as shown in Box II. where:

$$
\begin{array}{ll}
E_{21}=e_{1}+r_{1}, & E_{22}=e_{2}+r_{2}, \\
F_{21}=a_{1}+r_{1}, & F_{22}=b_{1}+\sum_{j=1}^{r_{1}} \log \left(\frac{1}{x_{1 j}}\right), \\
G_{21}=a_{2}+r_{2}, & G_{22}=b_{2}+\sum_{j=1}^{r_{2}} \log \left(\frac{1}{x_{2 j}}\right),
\end{array}
$$

$$
\begin{aligned}
H_{21}=c_{1}+r_{1}, \quad H_{21}=d_{1}+\sum_{j=1}^{r_{1}} x_{1 j}^{\alpha_{2}}, \\
I_{21}=c_{2}+r_{2}, \quad I_{22}=d_{2}+\sum_{j=1}^{r_{1}} x_{2 j}^{\alpha_{2}}, \\
J_{21}=\sum_{j=i}^{r_{1}} \log \left\{1-\lambda_{1}+2 \lambda_{1} \exp \left(-\frac{x_{1 j}^{\alpha_{1}}}{\beta_{1}}\right)\right\}, \\
J_{22}=\exp \left[\sum_{j=i}^{r_{2}} \log \left\{1-\lambda_{2}+2 \lambda_{2} \exp \left(-\frac{x_{2 j}^{\alpha_{2}}}{\beta_{2}}\right)\right\}\right] \\
K=1-p_{1} \exp \left(-\frac{T^{\alpha_{1}}}{\beta_{1}}\right)\left[1-\lambda_{1}+2 \lambda_{1} \exp \left(-\frac{T^{\alpha_{1}}}{\beta_{1}}\right)\right] \\
-\left(1-p_{1}\right) \exp \left(-\frac{T^{\alpha_{2}}}{\beta_{2}}\right) \\
{\left[1-\lambda_{2}+\lambda_{2} \exp \left(-\frac{T^{\alpha_{2}}}{\beta_{2}}\right)\right] . }
\end{aligned}
$$

The marginal posterior densities can be obtained by integrating out the nuisance parameters, for example, to derive the marginal density of parameter $\propto_{1}$, one proceeds as follows:

$$
\begin{array}{r}
g_{2}\left(\alpha_{1} \mid \mathbf{x}\right)=\int_{0}^{\infty} \int_{0}^{\infty} \int_{0}^{\infty} \int_{-1}^{1} \int_{-1}^{1} \int_{0}^{1} g_{2}(\Omega \mid \mathbf{x}) \\
d p_{1} d \lambda_{1} d \lambda_{2} d \beta_{1} d \beta_{2} d \alpha_{2}
\end{array}
$$

and vice versa.

$g_{I P}(\Omega \mid x)=$

$$
\begin{aligned}
& p_{1}^{E_{21}}\left(1-p_{1}\right)^{E_{22}-1} \alpha_{1}^{F_{21}-1} \exp \left(-\alpha_{1} F_{22}\right) \alpha_{2}^{G_{21}-1} \exp \left(-\alpha_{2} G_{22}\right) \frac{1}{\beta_{1}^{H}{ }_{21}+1} \exp \left(-\frac{H_{22}}{\beta_{1}}\right) \frac{1}{\beta_{2}^{I_{21}+1}} \exp \left(-\beta_{2} I_{22}\right) \\
& \exp \left(J_{21}\right) \exp \left(J_{22}\right)(K)^{n-r} \\
& \int_{0}^{\infty} \int_{0}^{\infty} \int_{0}^{\infty} \int_{0}^{\infty} \int_{-1}^{1} \int_{-1}^{1} p_{1}^{E} 21\left(1-p_{1}\right)^{E_{22}-1} \alpha_{1}^{F_{21}-1} \exp \left(-\alpha_{1} F_{22}\right) \alpha_{2}^{G_{21}-1} \exp \left(-\alpha_{2} G_{22}\right) \frac{1}{\beta_{1}^{H_{21}+1}} \exp \left(-\frac{H_{22}}{\beta_{1}}\right) \frac{1}{\beta_{2}^{I} 21+1} \exp \left(-\beta_{2} I_{22}\right) \\
& \int_{0} \int_{0} \int_{0} \int_{-1}^{\infty} \int_{-1} \exp \left(J_{21}\right) \exp \left(J_{22}\right)(K)^{n-r} d p_{1} d \lambda_{1} d \lambda_{2} d \alpha_{1} d \alpha_{2} d \beta_{1} d \beta_{2}
\end{aligned}
$$




\section{Bayes estimators and PRs under different loss functions}

In this section, derivation of the Bayes estimators and PRs under different loss functions are the main focus. The choice of a suitable loss function, i.e., symmetric or asymmetric, depends on the problem at hand, as there is no rule to select an appropriate loss function [30]. To estimate the unknown parameter in Bayesian, one must specify a loss function. A loss function $L(\theta, d) \geq 0$ is a function which enables one to estimate the unknown parameter by an estimator $\hat{d}$. The Bayes estimator is a posterior estimator which minimizes the posterior risk. The worth of a decision is measured by the expected loss, which is known as the posterior risk. If $\hat{d}$ is a Bayes estimator, then $\rho(\hat{d})$ is called the posterior risk [31], defined as:

$$
\rho(\hat{d})=E_{\theta \mid x}\{L(\theta, \hat{d})\}=\int L(\theta, \hat{d}) p(\theta \mid x) d \theta .
$$

One of the aims of this study is to suggest a suitable loss function for the parameter estimation of the TWD. To this end, three different loss functions, namely, the Squared Error Loss Function (SELF), Precautionary Loss Function (PLF) and Quadratic Loss Function (QLF), are used in this study. A loss function that yields the minimum posterior risk will be selected as the appropriate loss function. A brief discussion of these loss functions is given below.

The first loss function is the square error loss function, which is a symmetric loss function proposed by Gauss [32] and used by Legendre [33] to develop the least square theory. Further, it is also used in estimation problems. In fact, it is a quadratic deviation from the true parameter value. Mathematically, it is defined as:

$$
L(\beta, d)=(\beta-d)^{2},
$$

where $d$ is a decision that a statistician has to make in order to approximate an unknown $\beta$. This so called parameter is often used to summarize a posterior distribution.

The Bayes estimator and the posterior risk under SELF are:

$$
\begin{aligned}
& \hat{d}=E_{\beta \mid x}(\beta) \text { and } \\
& \rho(\hat{d})=E\left(\beta^{2} \mid x\right)-\{E(\beta \mid x)\}^{2}=\operatorname{Var}(\beta \mid x) .
\end{aligned}
$$

The PLF suggested by Norstrom [34] is an asymmetric loss function and defined as:

$$
L(\beta, d)=\frac{(\beta-d)^{2}}{d} .
$$

The Bayes estimator and the posterior risk under PLF are:

$$
\hat{d}=\left\{E\left(\beta^{2} \mid x\right\}^{\frac{1}{2}} \text { and } \rho(\hat{d})=2\left\{E\left(\beta^{2} \mid x\right\}^{\frac{1}{2}}-2 E(\beta \mid x) .\right.\right.
$$

The third loss function is the QLF, which is another asymmetric loss function and defined as:

$$
L(\beta, d)=\left(\frac{\beta-d}{\beta}\right)^{2} .
$$

The Bayes estimator and posterior risk for this loss function are:

$$
\hat{d}=\frac{E\left(\beta^{-1} \mid x\right)}{E\left(\beta^{-2} \mid x\right)} \text { and } \rho(\hat{d})=1-\frac{\left\{E\left(\beta^{-1} \mid x\right)\right\}^{2}}{E\left(\beta^{-2} \mid x\right)} .
$$

The details about these loss functions can be seen in $[35,36]$.

\subsection{Posterior summaries by MCMC technique} In the previous section, it is observed that the expressions of the posterior densities are in intractable form and cannot be solved directly. Thus, a numerical technique is adopted in order to find the posterior summaries, such as the mean and the quantiles. To this end, a MCMC technique is used, similar to Ali [36]. In particular, the Gibbs sampling [37] with metropolis hasting step $[38,39]$ is implemented. To obtain the posterior summaries using the MCMC approach, the posterior densities assuming the uniform and the informative priors can be written as:

$$
\begin{aligned}
& g_{U P}(\Omega \mid x) \propto f_{\alpha_{1}}\left(r_{1}+1, \sum_{j=1}^{r_{1}} \log \left(\frac{1}{x_{1 j}}\right)\right) \\
& \times f_{\alpha_{2}}\left(r_{2}+1, \sum_{j=1}^{r_{2}} \log \left(\frac{1}{x_{2 j}}\right)\right) \\
& \times f_{\beta_{1} \mid \alpha_{1}}\left(r_{1}-1, \sum_{i=1}^{r_{1}}\left(x_{1 i}^{\alpha_{1 i}}+(n-r) T^{\alpha_{1 i}}\right)\right. \\
& \times f_{\beta_{2} \mid \alpha_{2}}\left(r_{2}-1, \sum_{i=1}^{r_{2}}\left(x_{2 i}^{\alpha_{2}}+(n-r) T^{\alpha_{2 i}}\right)\right. \\
& \times f_{\lambda_{1}}\left(\exp \left[\sum_{i=1}^{r_{1}} \log \left\{1-\lambda_{1}+2 \lambda_{1} \exp \left(-\frac{x^{\alpha_{1}} 1 j}{\beta_{1}}\right)\right\}\right]\right) \\
& \times f_{\lambda_{\alpha_{2}}}\left(\exp \left[\sum_{i=1 i}^{r_{2}} \log \left\{1-\lambda_{2}+2 \lambda_{2} \exp \left(-\frac{x_{2 j}^{\alpha_{2}}}{\beta_{2}}\right)\right\}\right]\right) \\
& \left.g_{I P}(\Omega \mid x) \propto r_{2}+1, b_{2}+\sum_{j=1}^{r_{2}} \log \left(\frac{1}{x_{2 j}}\right)\right)
\end{aligned}
$$




$$
\begin{aligned}
& \times f_{\left.\beta_{1}\right|_{1} \alpha_{1}}\left(c_{1}+r_{1}, d_{1}+\sum_{i=1}^{r_{1}}\left(x_{1 i}^{\alpha_{1}}+(n-r) T^{\alpha_{1}}\right)\right. \\
& \times f_{\beta_{2} \mid \alpha_{2}}\left(c_{2}+r_{2} d_{2}+\sum_{i=1}^{r_{2}}\left(x_{2 i}^{\alpha_{2}}+(n-r) T^{\alpha_{2 i}}\right)\right. \\
& \times f_{\lambda_{1}}\left(\exp \left[\sum_{j=i}^{r_{1}} \log \left\{1-\lambda_{1}+2 \lambda_{1} \exp \left(-\frac{x_{1 i}^{\alpha_{1}}}{\beta_{1}}\right)\right\}\right]\right) \\
& \times f_{\lambda_{2}}\left(\exp \left[\sum_{j=i}^{r_{2}} \log \left\{1-\lambda_{2}+2 \lambda_{2} \exp \left(-\frac{x_{2 j}^{\alpha_{2}}}{\beta_{2}}\right)\right\}\right]\right)
\end{aligned}
$$

where $f_{\alpha_{1}}$ and $f_{\beta_{1} \mid \alpha_{1}}$ denote the PDFs of gamma and inverse gamma distributions while $f_{\lambda_{1}}$ is the PDFs of $\lambda$ for the first component. Similarly $f_{\alpha_{2}}, f_{\beta_{2} \mid \alpha_{2}}$ and $f_{\lambda_{2}}$ represent the PDFs of a gamma, inverse gamma and $\lambda$ for the second component. In order to obtain the BEs and their respective PRs, one proceeds as follows.

Random numbers from the TWD are generated by using the inverse integral transformation, i.e.:

$$
u_{i}=\left(1-e^{-\frac{x^{\alpha_{i}}}{\beta_{i}}}\right)\left(1-\lambda_{i}+\lambda_{i} e^{-\frac{x^{\alpha_{i}}}{\beta_{i}}}\right) \text {, }
$$

where $i=1,2$ and $u_{i} \sim$ uniform $(0,1)$. After simplification:

$$
x_{i}=\left\{-\beta_{i} \ln \left(\frac{1+\lambda_{i}-\sqrt{\left(1+\lambda_{i}\right)^{2}-4 \lambda_{i}} u_{i}}{2 \lambda_{i}}\right)\right\}^{\frac{1}{\alpha_{i}}}
$$

is obtained.

Thus, one can obtain the desired random sample by providing the required parameters. To generate the right censored data, one needs to fix $T$ and record units that are less than equal to the censoring time. The number of units that are greater than censoring time would be considered censored observations. To generate data from the mixture model, fix $p$ and generate uniform $(0,1)$. If the generated uniform random number is smaller than $p$, generate $X_{1}$ from $T W D\left(\alpha_{1}, \beta_{1}, \lambda_{1}\right)$, otherwise, from $T W D\left(\alpha_{2}, \beta_{2}, \lambda_{2}\right)$.

Next, to implement the MCMC for finding the posterior summaries, the following steps are proposed:

\section{Algorithm 1}

At the $i$ th step, repeat the following steps:

1. Generate:

$$
\begin{aligned}
& \alpha_{1 i} \sim f_{\alpha_{1}}=\text { Gamma }\left(r_{1}+1, \sum_{j=1}^{r_{1}} \log \left(\frac{1}{x_{1 j}}\right)\right), \\
& \beta_{1 i} \sim f_{\beta_{1} \mid \alpha_{1(i-1)}}=\text { Inverse }- \text { Gamma }
\end{aligned}
$$

$$
\begin{gathered}
\left(r_{1}-1, \sum_{i=1}^{r_{1}}\left(x_{1 i}^{\alpha_{1 i}}+(n-r) T^{\alpha_{1 i}}\right),\right. \\
\alpha_{2 i} \sim f_{\alpha_{2}}=\text { Gamma }\left(r_{2}+1, \sum_{j=1}^{r_{2}} \log \left(\frac{1}{x_{2 j}}\right)\right) \\
\beta_{2 i} \sim f_{\beta_{2} \mid \alpha_{1(i-1)}}=\text { Inverse }- \text { Gamma }\left(r_{2}-1,\right. \\
\sum_{i=1}^{r_{2}}\left(x_{2 i}^{\alpha_{2}}+(n-r) T^{\alpha_{2 i}}\right) .
\end{gathered}
$$

2. Take $Y_{1 i} \sim$ Uniform $(0,1)$ and $\mathrm{Y}_{2 \mathrm{i}} \sim$ Uniform $(0,1)$.

3. Generate $U \sim U$ iform $(0,1)$ and, $\lambda_{1 i}^{*}$ and $\lambda_{2 i}^{*}$ from Uniform $(-1,1)$.

4. Let:

$$
\begin{aligned}
& f_{1}^{(i)}=\exp \left[\sum _ { i = 1 } ^ { r _ { 1 } } \left\{\operatorname { l o g } \left(1-\lambda_{1}^{*(i-1)}+2 \lambda_{1}^{*(i-1)}\right.\right.\right. \\
& \left.\left.\left.\exp \left(-x_{1}^{\alpha_{1}^{(i)}} / \beta_{1}^{(i)}\right)\right)\right\}\right] \\
& f_{2}^{(i)}=\exp \left[\sum _ { i = 1 } ^ { r _ { 1 } } \left\{\operatorname { l o g } \left(1-Y_{1}^{(i)}+2 Y_{1}^{(i)}\right.\right.\right. \\
& \left.\left.\left.\exp \left(-x_{1}^{\alpha_{1}^{(i)}} / \beta_{1}^{(i)}\right)\right)\right\}\right] \\
& f_{3}^{(i)}=\exp \left[\sum _ { i = 1 } ^ { r _ { 2 } } \left\{\operatorname { l o g } \left(1-\lambda_{2}^{*(i-1)}+2 \lambda_{2}^{*(i-1)}\right.\right.\right. \\
& \left.\left.\left.\exp \left(-x_{2}^{\alpha_{2}^{(i)}} / \beta_{2}^{(i)}\right)\right)\right\}\right] \\
& f_{4}^{(i)}=\exp \left[\sum _ { i = 1 } ^ { r _ { 2 } } \left\{\operatorname { l o g } \left(1+Y_{2}^{(i)}-2 Y_{2}^{(i)}\right.\right.\right. \\
& \left.\left.\left.\exp \left(-x_{2}^{\alpha_{2}^{(i)}} / \beta_{2}^{(i)}\right)\right)\right\}\right] \\
& \rho_{1}^{(i)}=\min \left[1, \frac{f_{1}^{(i)}\left\{\operatorname{dunif}\left(\lambda_{1}^{*(i-1)}, 0,1\right)\right\}}{f_{2}^{(i)}\left\{\operatorname{dunif}\left(\lambda_{1}^{*(i-1)}, 0,1\right)\right\}}\right] \\
& \rho_{2}^{(i)}=\min \left[1, \frac{f_{3}^{(i)}\left\{\operatorname{dunif}\left(\lambda_{2}^{*(i-1)}, 0,1\right)\right\}}{f_{4}^{(i)}\left\{\operatorname{dunif}\left(\lambda_{2}^{*(i-1)}, 0,1\right)\right\}}\right]
\end{aligned}
$$

5. Select,

$$
\lambda_{1}^{(i)}=\lambda_{1}^{*(i)} \begin{cases}\lambda_{1}^{*(\mathrm{i})}=\lambda_{1}^{*(i-1)} & \text { if } \rho_{1}^{(i)}>U^{(i)} \\ \lambda_{1}^{*(\mathrm{i})}=Y_{1}^{*(i-1)} & \text { otherwise }\end{cases}
$$




$$
\lambda_{2}^{(i)}=\lambda_{2}^{*(i)} \begin{cases}\lambda_{2}^{*(\mathrm{i})}=\lambda_{2}^{*(i-1)} & \text { if } \rho_{2}^{(i)}>U^{(i)} \\ \lambda_{2}^{*(\mathrm{i})}=Y_{2}^{*(i-1)} & \text { otherwise }\end{cases}
$$

6. Repeat steps 1-6, $N$-times to obtain $\left(\alpha_{1}, \beta_{1}, \lambda_{1}\right)$, $\left(\alpha_{2}, \beta_{2}, \lambda_{2}\right), \ldots\left(\alpha_{N}, \beta_{N}, \lambda_{N}\right)$ and discard first $M$ observations as the burn-in period.

7. The approximate values of $\hat{\alpha}_{1}, \hat{\alpha}_{2}, \hat{\beta}_{1}, \hat{\beta}_{2}, \hat{\lambda}_{1}$, and $\hat{\lambda}_{2}$ are:

$$
\begin{aligned}
& \hat{\alpha}_{1}=\frac{\sum_{i=M+1}^{N} \alpha_{1 i} k\left(\alpha_{1 i}, \alpha_{2 i}, \beta_{1 i}, \beta_{2 i}, \lambda_{1 i}, \lambda_{2 i}, p_{1 i}\right)}{\sum_{i=M+1}^{N} k\left(\alpha_{1 i}, \alpha_{2 i}, \beta_{1 i}, \beta_{2 i}, \lambda_{1 i}, \lambda_{2 i}, p_{1 i}\right)}, \\
& \hat{\beta}_{1}=\frac{\sum_{i=M+1}^{N} \beta_{1 i} k\left(\alpha_{1 i}, \alpha_{2 i}, \beta_{1 i}, \beta_{2 i}, \lambda_{1 i}, \lambda_{2 i}, p_{1 i}\right)}{\sum_{i=M+1}^{N} k\left(\alpha_{1 i}, \alpha_{2 i}, \beta_{1 i}, \beta_{2 i}, \lambda_{1 i}, \lambda_{2 i}, p_{1 i}\right)}, \\
& \hat{\lambda}_{1}=\frac{\sum_{i=M+1}^{N} \lambda_{1}^{(i)} k\left(\alpha_{1 i}, \alpha_{2 i}, \beta_{1 i}, \beta_{2 i}, \lambda_{1 i}, \lambda_{2 i}, p_{1 i}\right)}{\sum_{i=M+1}^{N} k\left(\alpha_{1 i}, \alpha_{2 i}, \beta_{1 i}, \beta_{2 i}, \lambda_{1 i}, \lambda_{2 i}, p_{1 i}\right)}, \\
& \hat{\alpha}_{2}=\frac{\sum_{i=M+1}^{N} \alpha_{2 i} k\left(\alpha_{1 i}, \alpha_{2 i}, \beta_{1 i}, \beta_{2 i}, \lambda_{1 i}, \lambda_{2 i}, p_{1 i}\right)}{\sum_{i=M+1}^{N} k\left(\alpha_{1 i}, \alpha_{2 i}, \beta_{1 i}, \beta_{2 i}, \lambda_{1 i}, \lambda_{2 i}, p_{1 i}\right)}, \\
& \hat{\beta}_{2}=\frac{\sum_{i=M+1}^{N} \beta_{2 i} k\left(\alpha_{1 i}, \alpha_{2 i}, \beta_{1 i}, \beta_{2 i}, \lambda_{1 i}, \lambda_{2 i}, p_{1 i}\right)}{\sum_{i=M+1}^{N} k\left(\alpha_{1 i}, \alpha_{2 i}, \beta_{1 i}, \beta_{2 i}, \lambda_{1 i}, \lambda_{2 i}, p_{1 i}\right)}, \\
& \sum_{i=M+1}^{N} \lambda_{2}^{(i)} k\left(\alpha_{1 i}, \alpha_{2 i}, \beta_{1 i}, \beta_{2 i}, \lambda_{1 i}, \lambda_{2 i}, p_{1 i}\right) \\
& \left.\hat{\lambda}_{1 i}, \alpha_{2 i}, \beta_{1 i}, \beta_{2 i}, \lambda_{1 i}, \lambda_{2 i}, p_{1 i}\right)
\end{aligned}
$$

In the next section, the performance of Bayes estimators is evaluated on the basis of PR for the informative and the noninformative priors under different loss functions.

\section{A simulation study and some comparisons}

In this section, a comprehensive simulation study has been carried out to assess the performance of the Bayes estimators assuming different loss functions, sample sizes, mixing weights, parameter values, and censoring rates using non-informative and informative priors in terms of PRs. Samples of sizes $n=20,40,60$, and 100 have been generated by the inverse transformation methods from the two-component mixture of the TWD with parameters $\alpha_{1}, \alpha_{2}, \beta_{1}, \beta_{2}, \lambda_{1}$ and $\lambda_{2}$, such that $(1.5,2,2,1.4,0.4,0.5),(2.5,3,1.6,1.3,0.3,0.4)$, $(2,1.5,1.4,1.3,0.4,0.6)$ and probabilistic mixing weights $p_{1} \in\{(0.3,0.5,0.7)\}$. The $p_{1} n$ observations were generated randomly from the first component density $f_{1}\left(x_{1} ; \alpha_{1}, \beta_{1}, \lambda_{1}\right)$ and the remaining $\left(1-p_{1}\right) n$ were generated from the second component density $f_{2}\left(x_{2} ; \alpha_{2}, \beta_{2}, \lambda_{2}\right)$, respectively. For a fixed sample size, test termination time, the aforementioned algorithm was used, and the BEs and PRs were computed using the UP and the IP under the SELF, PLF and QLF. It is worth mentioning that right censoring was considered; all observations greater than fixed censoring time $T$ were considered censored. In each case, only failure items can be identified to be a member of either subpopulation-I or subpopulation-II of the mixture. In this study, different censoring rates were considered, for example, $20 \%$ and $40 \%$, to evaluate their impact on the Bayes estimators. For each of the combinations of parameters, different sample sizes and censoring rates, the steps of the algorithm are repeated $N=10,000$ times using $\mathrm{R}$ software, $\mathrm{R}$ [40]. The simulated results are then averaged by considering $M=2000$ as the burn-in period. The simulated BEs and the PRs using the UP and IP under SELF, PLF and QLF are tabulated in Tables 1, A.1 and A.2 (Tables A.1 and A.2 given in Appendix A), where the PRs have been presented in parentheses below the BEs.

The simulation study indicates that the estimated values of each parameter converge to the true value by increasing the sample size and magnitude of the corresponding PRs decreases at a fixed test termination time. This pattern is not restricted to any specific loss function or prior but observed for all the considered loss functions. The simulation study gives some interesting features of the BEs. By comparing the results of $20 \%$ and $40 \%$ censored rates, one can easily observe that the PRs for $20 \%$ censoring are smaller than $40 \%$ censoring, as shown in Tables 1, A.1 and A.2. The reason for this is the availability of more information for $20 \%$ censored data than $40 \%$ censored data; and due to this, PRs of $20 \%$ are smaller than those of $40 \%$. Furthermore, a direct effect of censoring rate on the posterior risk was observed, i.e., the posterior risk increased as the censoring rate increased. From Tables 1, A.1 and A.2, it is noticed that the IP is more accurate than the UP. Also, the convergence of $\mathrm{BEs}$ to the nominal value was observed to be faster in the case of IP than the UP for all assumed loss functions.

Next, the performance of different loss functions is compared. From Table 1, it can be observed that the BEs are found to be more efficient for the first set of parameter values under the IP as compared to the UP under SELF than PLF and QLF. In the case of $20 \%$ censoring rate and assuming IP, the 
Table 1. Bayes Estimates (BEs) of mixture for two components of Transmuted Weibull Distribution (TWD) along with Posterior Risks (PRs) (in parentheses) under Uniform Prior (UP) and Informative Prior (IP).

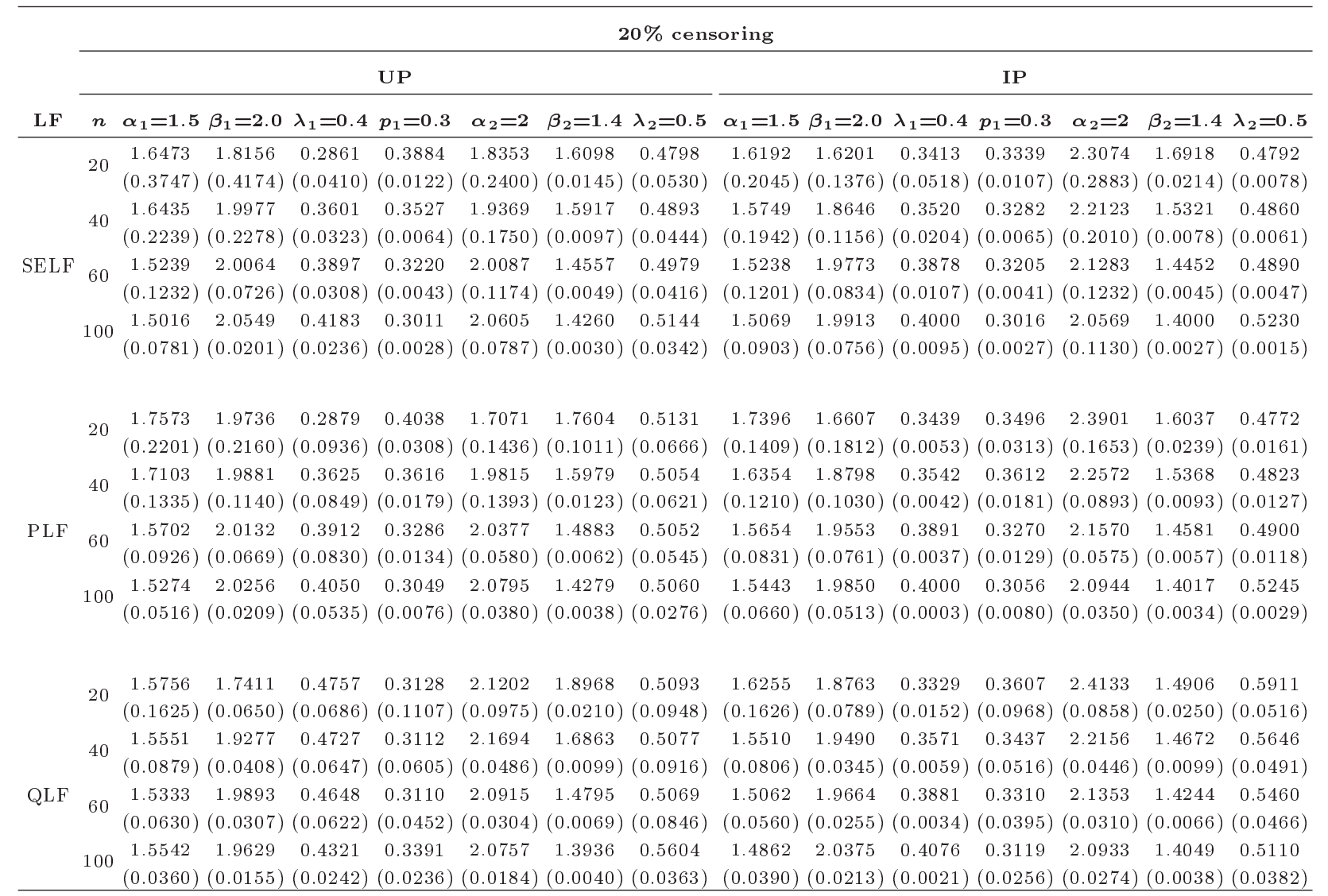

$40 \%$ censoring

UP IP

$\begin{array}{lllllllllllllllllllll}\mathrm{LF} & n & \alpha_{1}=1.5 & \beta_{1}=2.0 & \lambda_{1}=0.4 & p_{1}=0.3 & \alpha_{2}=2 & \beta_{2}=1.4 & \lambda_{2}=0.5 & \alpha_{1}=1.5 & \beta_{1}=2.0 & \lambda_{1}=0.4 & p_{1}=0.3 & \alpha_{2}=2 & \beta_{2}=1.4 & \lambda_{2}=0.5\end{array}$

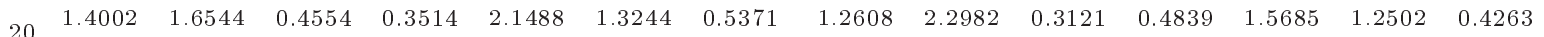
$(0.3757)(0.4189)(0.0527)(0.0165)(0.2722)(0.0923)(0.0576)(0.2052)(0.1584)(0.0652)(0.0151)(0.2947)(0.0495)(0.0137)$

$\begin{array}{llllllllllllll}1.4778 & 1.7801 & 0.4378 & 0.3434 & 2.1245 & 1.3602 & 0.5225 & 1.3159 & 1.6476 & 0.3495 & 0.4194 & 1.9599 & 1.3401 & 0.4417\end{array}$ $(0.2384)(0.2854)(0.0458)(0.0084)(0.2019)(0.0634)(0.0569)(0.1949)(0.1248)(0.0212)(0.0087)(0.2252)(0.0356)(0.0107)$

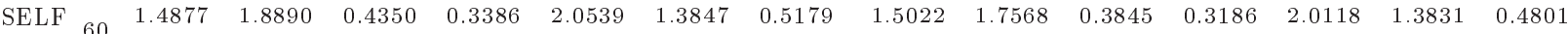
$(0.1429)(0.1056)(0.0360)(0.0057)(0.1229)(0.0263)(0.0544)(0.1392)(0.1071)(0.0123)(0.0050)(0.1426)(0.0102)(0.0076)$

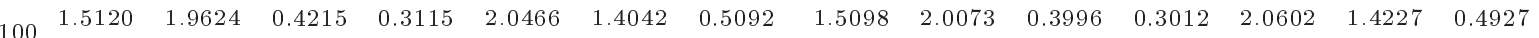
(0.0873) (0.0979) (0.0245) (0.0038) (0.0933) (0.0104) (0.0428) (0.0974) (0.0960)(0.0108)(0.0032)(0.1292) (0.0098)(0.0053)

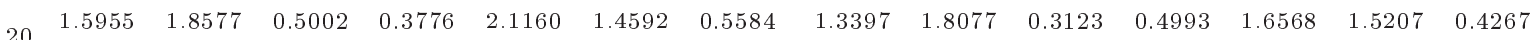
(0.2404) (0.3787) (0.0595) (0.0325) (0.2776) (0.1249) (0.1025) (0.1578) (0.2189) (0.0098) (0.0338) (0.1766) (0.0410) (0.0286)

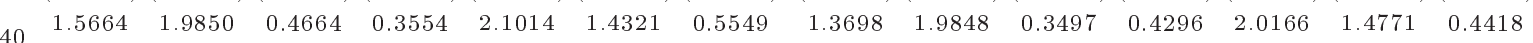
0 (0.1571) (0.3558) (0.0978) (0.0341) (0.1827) (0.0637) (0.0648) (0.1379) (0.1744) (0.0075) (0.0205) (0.1133) (0.0349) (0.0225)

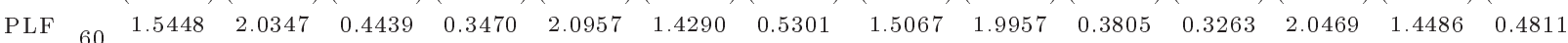
$(0.1141)(0.0912)(0.0860)(0.0268)(0.0834)(0.0286)(0.0644)(0.0917)(0.0568)(0.0061)(0.0193)(0.0702)(0.0110)(0.0214)$

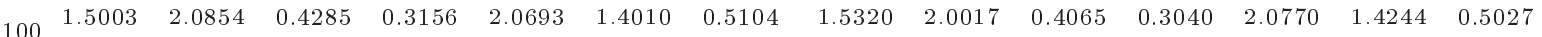
(0.0766) (0.0460) (0.0840) (0.0190) (0.0454) (0.0115) (0.0424) (0.0843) (0.0453) (0.0045) (0.0155) (0.0335) (0.0073) (0.0185)

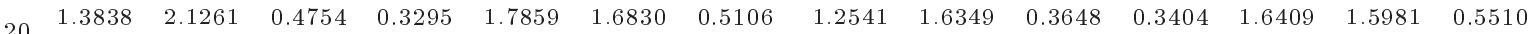
(0.1998) (0.1244) (0.0806) (0.1416) (0.1477) (0.0736) (0.0985) (0.1778) (0.1201) (0.0155) (0.1098) (0.1258) (0.0702) (0.0576)

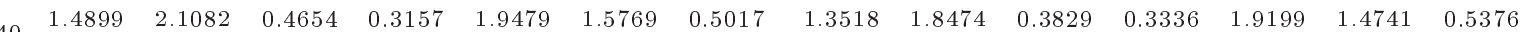
$(0.1128)(0.0851)(0.0691)(0.0718)(0.0679)(0.0301)(0.0960)(0.0942)(0.0735)(0.0080)(0.0670)(0.0597)(0.0353)(0.0538)$

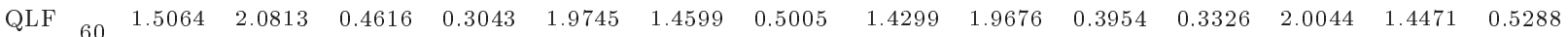
$(0.0788)(0.0731)(0.0645)(0.0599)(0.0414)(0.0218)(0.0927)(0.0770)(0.0611)(0.0041)(0.0457)(0.0444)(0.0244)(0.0514)$

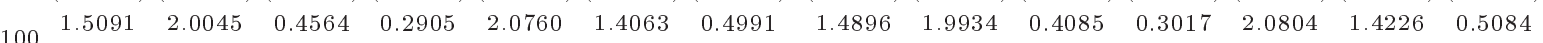
100 (0.0528) (0.0578) (0.0516) (0.0349) (0.0280) (0.0103) (0.0792) (0.0452) (0.0516) (0.0035) (0.0331) (0.0246) (0.0176) (0.0404) 
transmuted parameters $\left(\lambda_{1}, \lambda_{2}\right)$ of both components are over estimated under the assumed loss functions. It is clear from Table A.1 that if $\alpha_{1}<\alpha_{2}$, the estimated values of the shape and the transmuted parameters are over-estimated under the considered loss functions for $20 \%$ and $40 \%$ censoring rates. It is also noticed that IP gives us more precise estimates using the SELF for the second set of parametric values by considering $20 \%$ censoring rate than the PLF and the QLF. From Table A.2, it is clear that IP produced more accurate estimates for all parameters under QLF when $40 \%$ censoring was considered. Also, for the third set of parameters, if $\alpha_{1}>\alpha_{2}$ the estimated values of the shape and the scale parameters are overestimated for the first component, while under-estimated for the second component under SELF. Among all sets of parametric values, the QLF is the most suitable for both $20 \%$ and $40 \%$ censoring rates (cf. Tables 1 , A.1 and A.2). In the case of $20 \%$ censoring rate, to estimate the mixing component parameter $p_{1}$, the SELF is observed to be more superior than the other two loss functions.

\section{Bayesian Credible Intervals (BCI)}

The goal of this section is to obtain the BCI for the unknown parameters. The BCI are obtained by utilizing the marginal posterior densities of the parameter of interest. A 95\% Bayesian credible interval (sometimes also known as the posterior interval) is analogous to the familiar $95 \%$ frequentist confidence interval which provides a range of likely values for a parameter [41].

Given the data, the $95 \%$ credibility interval $\left(\mu_{L}, \mu_{U}\right)$ includes the true $\mu$ with probability $95 \%$, whereas the frequentist intervals may include it or not, i.e., its inclusion probability is either zero or one. According to Eberly and Casella [42], the 100 (1-k) \% credible intervals can be obtained by solving the following equation:

$$
\int_{0}^{L} g(\alpha \mid x) d \alpha=\frac{k}{2}=\int_{U}^{\infty} g(\alpha \mid x) d \alpha,
$$

where $g(\alpha \mid x)$ is the posterior distribution of $\alpha$ given data, $L$ and $U$ denote the lower and upper limits of the credible interval, respectively, and $k$ is the significance level.

In the case of interval estimation (Tables 2 and A.3-A.4), it is observed that the width of $95 \%$ credible intervals reduces as the sample size increases. Also, all the credible intervals contain the nominal value of the respective unknown parameters. The least widths for BCI has been observed for the $20 \%$ censoring rate rather than the $40 \%$. The reason for this is that there is more information in the case of a small censoring rate rather than for the large one.

\section{Real life application}

In this section, two real-life data sets are analyzed in order to illustrate the practicality of the mixture distribution in practice. For the sake of space, the results of the first data set are presented here, whereas the second data set is discussed in Appendix A.

\subsection{Real data Set-I: Hepatitis analysis}

A real data set was analyzed to illustrate the methodology discussed in the previous section. The data set has been collected from the Combined Military Hospital (CMH) Rawalpindi, Pakistan. In order to show the usefulness of the proposed mixture distribution, Bayesian analysis was performed on the life time data of hepatitis B \& C patients to estimate the unknown parameters, assuming UP and IP under SELF, PLF and QLF. This data set consists of 80 values regarding recovery time period (in days) of hepatitis (B \& C) patients treated with antiviral medications such as lamivudine for hepatitis B or ribavirin (trade name Copegus, Rebetol, Ribasphere) for hepatitis C. For the sake of privacy, admission date, discharge date and number of recovery days from 2016 to 2017 are reported. Since the patients have two different types of hepatitis, the data can easily be divided into two subpopulations. In Table 3 , the hepatitis $\mathrm{B}$ patients have been classified into subpopulation-1, while the hepatitis $\mathrm{C}$ patients into subpopulation 2. The appropriateness of the twocomponent mixture of the TWD is tested against the Weibull and transmuted Weibull by using $\chi^{2}$ - statistic and the $P$-value is 0.4559 . Thus, it is evident that the mixture distribution is a good fit against the other assumed models.

For the analysis, the data (Table 3 ) was categorized into two groups with probability mixing weight $p_{1}=0.5$ and $T=30$. The reason for considering $T=30$ is that if a patient does not recover within one month then he is referred to the CMH Lahore. The summary of the data is as follows:

$$
\begin{aligned}
& n_{1}=40, \quad r_{1}=40, \quad r_{2}=8, \quad p_{1}=0.5, \quad \sum_{j=1}^{r_{1}} x_{1 j}=680, \\
& \sum_{j=1}^{r_{1}} \log \left(1 / x_{1 j}\right)=-112.88, \\
& n_{2}=40, \quad r_{2}=19, \quad \sum_{j=1}^{r_{2}} x_{2 j}=523, \\
& \sum_{j=1}^{r_{2}} \log \left(1 / x_{2 j}\right)=-62.96 .
\end{aligned}
$$

The BEs, the PRs (in parenthesis) and interval 
Table 2. 95\% Bayesian credible intervals of mixture for two components of Transmuted Weibull Distribution (TWD) using Uniform Prior (UP) and Informative Prior (IP) with hyperparameters are $a_{1}=0.5, a_{2}=1.5, b_{1}=1, b_{2}=1$, $c_{1}=0.5, c_{2}=1, d_{1}=1, d_{2}=2, e_{1}=0.5, e_{2}=1, l_{1}=0.1$, and $l_{2}=0.2$.

\begin{tabular}{|c|c|c|c|c|c|c|}
\hline \multirow{2}{*}{$\begin{array}{c}\text { Censoring } \\
\text { rate }\end{array}$} & \multirow[t]{2}{*}{ Size } & \multirow[t]{2}{*}{ Parameters } & \multicolumn{2}{|c|}{ UP } & \multicolumn{2}{|c|}{ IP } \\
\hline & & & Lower limit & Upper limit & Lower limit & Upper limit \\
\hline \multirow{8}{*}{$20 \%$} & \multirow{14}{*}{20} & $\alpha_{1}=1.5$ & 1.0193 & 10.8590 & 1.5076 & 6.8866 \\
\hline & & $\alpha_{2}=2.0$ & 1.0457 & 9.9829 & 1.4628 & 6.0349 \\
\hline & & $\beta_{1}=2.0$ & 0.6648 & 6.4781 & 0.8483 & 3.0799 \\
\hline & & $\beta_{2}=1.4$ & 0.6321 & 4.8065 & 0.6979 & 2.6335 \\
\hline & & $\lambda_{1}=0.4$ & 0.3482 & 0.9361 & 0.3451 & 0.4481 \\
\hline & & $\lambda_{2}=0.5$ & 0.4184 & 0.9681 & 0.4165 & 0.6683 \\
\hline & & $p_{1}=0.3$ & 0.2273 & 3.0082 & 0.2031 & 1.8047 \\
\hline & & $\alpha_{1}=1.5$ & 1.0237 & 10.9073 & 1.5085 & 7.7015 \\
\hline \multirow{6}{*}{$40 \%$} & & $\alpha_{2}=2.0$ & 1.0469 & 10.5421 & 1.4895 & 7.9162 \\
\hline & & $\beta_{1}=2.0$ & 0.7477 & 6.8479 & 0.8618 & 8.2817 \\
\hline & & $\beta_{2}=1.4$ & 0.6688 & 6.0649 & 0.7412 & 2.8065 \\
\hline & & $\lambda_{1}=0.4$ & 0.3513 & 0.9430 & 0.3541 & 0.4507 \\
\hline & & $\lambda_{2}=0.5$ & 0.4283 & 0.9804 & 0.4254 & 0.6784 \\
\hline & & $p_{1}=0.3$ & 0.2489 & 3.0695 & 0.2667 & 2.2364 \\
\hline \multirow{6}{*}{$20 \%$} & \multirow{14}{*}{40} & $\alpha_{1}=1.5$ & 0.9370 & 8.1197 & 0.9224 & 6.7606 \\
\hline & & $\alpha_{2}=2.0$ & 1.0357 & 9.6256 & 0.9959 & 5.4217 \\
\hline & & $\beta_{1}=2.0$ & 0.5157 & 6.4623 & 0.5905 & 2.8047 \\
\hline & & $\beta_{2}=1.4$ & 0.4350 & 4.5378 & 0.4692 & 2.2673 \\
\hline & & $\lambda_{1}=0.4$ & 0.3396 & 0.8948 & 0.3046 & 0.4225 \\
\hline & & $\lambda_{2}=0.5$ & 0.4034 & 0.9466 & 0.4207 & 0.6655 \\
\hline \multirow{8}{*}{$40 \%$} & & $p_{1}=0.3$ & 0.1524 & 2.3285 & 0.1541 & 1.0640 \\
\hline & & $\alpha_{1}=1.5$ & 0.9497 & 9.3651 & 0.9588 & 6.6882 \\
\hline & & $\alpha_{2}=2.0$ & 1.0404 & 10.2397 & 0.9995 & 6.6487 \\
\hline & & $\beta_{1}=2.0$ & 0.5951 & 6.4667 & 0.6371 & 3.7396 \\
\hline & & $\beta_{2}=1.4$ & 0.4452 & 4.9274 & 0.4770 & 2.3125 \\
\hline & & $\lambda_{1}=0.4$ & 0.3443 & 0.9032 & 0.3059 & 0.4412 \\
\hline & & $\lambda_{2}=0.5$ & 0.3976 & 0.9474 & 0.4217 & 0.6717 \\
\hline & & $p_{1}=0.3$ & 0.1660 & 2.6785 & 0.1799 & 2.0907 \\
\hline \multirow{7}{*}{$20 \%$} & \multirow{14}{*}{60} & $\alpha_{1}=1.5$ & 0.6360 & 7.7392 & 0.5754 & 6.5649 \\
\hline & & $\alpha_{2}=2.0$ & 0.6404 & 8.8064 & 0.7967 & 5.1786 \\
\hline & & $\beta_{1}=2.0$ & 0.3972 & 5.8619 & 0.4299 & 2.5934 \\
\hline & & $\beta_{2}=1.4$ & 0.3200 & 3.8016 & 0.3524 & 1.9025 \\
\hline & & $\lambda_{1}=0.4$ & 0.3315 & 0.8822 & 0.3023 & 0.4146 \\
\hline & & $\lambda_{2}=0.5$ & 0.3923 & 0.9396 & 0.4139 & 0.5786 \\
\hline & & $p_{1}=0.3$ & 0.1135 & 2.3008 & 0.1326 & 1.0598 \\
\hline \multirow{7}{*}{$40 \%$} & & $\alpha_{1}=1.5$ & 0.7982 & 8.8417 & 0.5820 & 6.5996 \\
\hline & & $\alpha_{2}=2.0$ & 0.6982 & 9.5806 & 0.8052 & 5.7471 \\
\hline & & $\beta_{1}=2.0$ & 0.4086 & 6.3806 & 0.4332 & 3.5954 \\
\hline & & $\beta_{2}=1.4$ & 0.3983 & 4.5960 & 0.3614 & 2.2963 \\
\hline & & $\lambda_{1}=0.4$ & 0.3360 & 0.8923 & 0.3053 & 0.4411 \\
\hline & & $\lambda_{2}=0.5$ & 0.3966 & 0.9426 & 0.4192 & 0.5992 \\
\hline & & $p_{1}=0.3$ & 0.1291 & 2.6386 & 0.1457 & 1.8416 \\
\hline
\end{tabular}


Table 2. $95 \%$ Bayesian credible intervals of mixture for two components of Transmuted Weibull Distribution (TWD) using Uniform Prior (UP) and Informative Prior (IP) with hyperparameters are $a_{1}=0.5, a_{2}=1.5, b_{1}=1, b_{2}=1$, $c_{1}=0.5, c_{2}=1, d_{1}=1, d_{2}=2, e_{1}=0.5, e_{2}=1, l_{1}=0.1$, and $l_{2}=0.2$ (continued).

\begin{tabular}{|c|c|c|c|c|c|c|}
\hline \multirow{2}{*}{$\begin{array}{c}\text { Censoring } \\
\text { rate }\end{array}$} & \multirow[t]{2}{*}{ Size } & \multirow[t]{2}{*}{ Parameters } & \multicolumn{2}{|c|}{ UP } & \multicolumn{2}{|c|}{ IP } \\
\hline & & & Lower limit & Upper limit & Lower limit & Upper limit \\
\hline \multirow{6}{*}{$20 \%$} & \multirow{14}{*}{100} & $\alpha_{1}=1.5$ & 0.2180 & 5.9429 & 0.3957 & 5.9407 \\
\hline & & $\alpha_{2}=2.0$ & 0.2834 & 8.0151 & 0.5317 & 5.1241 \\
\hline & & $\beta_{1}=2.0$ & 0.2475 & 5.5634 & 0.3043 & 2.2083 \\
\hline & & $\beta_{2}=1.4$ & 0.1861 & 3.7751 & 0.2424 & 1.9010 \\
\hline & & $\lambda_{1}=0.4$ & 0.3280 & 0.8779 & 0.3013 & 0.4110 \\
\hline & & $\lambda_{2}=0.5$ & 0.3918 & 0.9318 & 0.4120 & 0.5763 \\
\hline \multirow{8}{*}{$40 \%$} & & $p_{1}=0.3$ & 0.0698 & 1.8032 & 0.0960 & 1.0038 \\
\hline & & $\alpha_{1}=1.5$ & 0.2792 & 5.9633 & 0.4002 & 5.9556 \\
\hline & & $\alpha_{2}=2.0$ & 0.2931 & 8.2385 & 0.5459 & 5.5638 \\
\hline & & $\beta_{1}=2.0$ & 0.2506 & 6.3151 & 0.3174 & 3.0312 \\
\hline & & $\beta_{2}=1.4$ & 0.2186 & 4.5808 & 0.2532 & 2.1005 \\
\hline & & $\lambda_{1}=0.4$ & 0.3314 & 0.8806 & 0.3018 & 0.4286 \\
\hline & & $\lambda_{2}=0.5$ & 0.3981 & 0.9417 & 0.4130 & 0.5898 \\
\hline & & $p_{1}=0.3$ & 0.0702 & 2.0866 & 0.1086 & 1.5733 \\
\hline
\end{tabular}

estimates for the parameters of the subject distribution are obtained assuming UP and IP under SELF, PLF and QLF.

Tables 4 and 5 contain the Bayesian estimation of parameters of the mixture of the TWD. Examination of the results confirms the previous conclusion that the BEs and credible intervals based on IP under SELF provide comparatively better results than the UP for estimating component parameters. Further, it is noticed that the estimates have a minimum amount of PRs for $20 \%$ censoring rate rather than for the $40 \%$ for both priors and under assumed loss functions.

\section{Conclusion}

In this article, a two-component mixture of the Transmuted Weibull Distribution (TWD) was introduced and its parameters were estimated using the Bayesian method. An extensive simulation study is conducted to compare and highlight some important and interesting properties of the Bayes estimators of the twocomponent mixture of the TWD using noninformative (uniform) and informative (gamma) priors under Squared Error Loss Function (SELF), Percautionary Loss Function (PLF), and Quadratic Loss Function (QLF). First, the expression for posterior densities was derived and it was noticed that the densities were not in closed forms. Thus, a Markov Chain Monte Carlo (MCMC) technique was proposed to obtain the poste- rior summaries. The second objective of this study was the choice of an appropriate loss function and prior for the estimation of the mixture's parameters assuming type-I right censored data with different sample sizes and test termination times. To this end, different posterior summaries were obtained, like Bayes estimates and their respective posterior risk, and Bayesian credible intervals assuming different sample sizes and test termination times. Two different censoring rates, i.e., $20 \%$ and $40 \%$, were considered. From Tables 1, A.1 and A.2, it is clear that the estimated values become very close to the nominal value of the parameters and the Posterior Risks (PRs) decreased with the increase of sample size. Thus, simulated results follow the consistency property. It is also observed that the $40 \%$ censoring rate has a larger amount of PRs than the $20 \%$ censored data. Furthermore, the results obtained from the real-life data sets showed the same pattern, which confirmed that the proposed MCMC algorithm performed well to estimate the unknown parameters in the Bayesian framework. The PLF was observed the most preferable choice for the estimation of mixing component (proportion) than the SELF and the QLF. Also, the IP is a more efficient prior for estimating the shape and the scale parameters. To show the application of the proposed model, two real life data sets have been analyzed, and its appropriateness was tested through $\chi^{2}$ - statistic. In the future, a truncated mixture can be studied. 
Table 3. Real life data of the survival time (in days) of hepatitis (B \& C) Patients.

\begin{tabular}{|c|c|c|c|c|c|}
\hline \multicolumn{3}{|c|}{ Disease (hepatitis B) } & \multicolumn{3}{|c|}{ Disease (hepatitis C) } \\
\hline Admit date & Discharge date & $\begin{array}{c}\text { No of recovery } \\
\text { days }\end{array}$ & Admit date & Discharge date & $\begin{array}{c}\text { No of recovery } \\
\text { days }\end{array}$ \\
\hline 02-01-2016 & $16-01-2016$ & 15 & 02-01-2016 & $30-01-2016$ & 29 \\
\hline 03-02-2016 & $18-02-2016$ & 16 & 06-01-2016 & 04-02-2016 & 30 \\
\hline 03-03-2016 & $19-03-2016$ & 17 & 03-02-2016 & 01-03-2016 & 28 \\
\hline 04-03-2016 & 18-03-2016 & 15 & 06-04-2016 & 04-05-2016 & 29 \\
\hline 05-04-2016 & $21-04-2016$ & 17 & $16-04-2016$ & $16-05-2016$ & 31 \\
\hline 08-05-2016 & 24-05-2016 & 17 & 02-05-2016 & $31-05-2016$ & 30 \\
\hline $10-05-2016$ & $30-05-2016$ & 21 & 08-06-2016 & 10-07-2016 & 33 \\
\hline $12-06-2016$ & $30-06-2016$ & 19 & $15-06-2016$ & $16-06-2016$ & 32 \\
\hline 03-07-2016 & $19-07-2016$ & 17 & $21-07-2016$ & $20-08-2016$ & 30 \\
\hline $06-07-2016$ & $19-07-2016$ & 14 & $22-07-2016$ & $18-08-2016$ & 27 \\
\hline 01-08-2016 & $16-08-2016$ & 15 & $18-08-2016$ & $16-09-2016$ & 30 \\
\hline $22-08-2016$ & 07-09-2016 & 17 & $28-08-2016$ & 24-09-2016 & 28 \\
\hline 09-09-2016 & $28-09-2016$ & 20 & $28-08-2016$ & 26-09-2016 & 30 \\
\hline $15-09-2016$ & 02-10-2016 & 18 & 02-09-2016 & 02-10-2016 & 31 \\
\hline 04-10-2016 & $20-10-2016$ & 17 & $10-09-2016$ & $12-10-2016$ & 33 \\
\hline $28-10-2016$ & $16-11-2016$ & 20 & $25-09-2016$ & $22-10-2016$ & 28 \\
\hline $16-11-2016$ & 03-12-2016 & 19 & $15-10-2016$ & $16-11-2016$ & 33 \\
\hline $25-11-2016$ & $16-12-2016$ & 23 & $22-10-2016$ & $23-11-2016$ & 33 \\
\hline $12-12-2016$ & $26-12-2016$ & 14 & $28-10-2016$ & $24-11-2016$ & 27 \\
\hline 09-01-2017 & $18-01-2017$ & 10 & 07-11-2016 & $04-12-2016$ & 28 \\
\hline $13-02-2017$ & $28-02-2017$ & 16 & $15-12-2016$ & $16-12-2016$ & 32 \\
\hline $18-03-2017$ & 29-03-2017 & 12 & $24-12-2016$ & 21-01-2017 & 29 \\
\hline 24-03-2017 & 08-04-2017 & 16 & 02-01-2017 & $30-01-2017$ & 29 \\
\hline 05-04-2017 & 21-04-2017 & 17 & 08-01-2017 & $06-02-2017$ & 30 \\
\hline 08-05-2017 & $24-05-2017$ & 17 & 04-02-2017 & 01-03-2017 & 24 \\
\hline $12-05-2017$ & $30-05-2017$ & 19 & $16-04-2017$ & $10-05-2017$ & 25 \\
\hline $14-06-2017$ & $30-06-2017$ & 17 & $18-04-2017$ & $16-05-2017$ & 29 \\
\hline 03-07-2017 & $18-07-2017$ & 16 & 01-05-2017 & $31-05-2017$ & 31 \\
\hline 08-07-2017 & $22-07-2017$ & 15 & $05-06-2017$ & 08-07-2016 & 34 \\
\hline 01-08-2017 & $16-08-2017$ & 16 & $15-06-2017$ & $13-07-2017$ & 29 \\
\hline $24-08-2017$ & $11-09-2017$ & 19 & $20-07-2017$ & $14-08-2017$ & 26 \\
\hline $19-09-2017$ & 04-10-2017 & 16 & $21-07-2017$ & $15-08-2017$ & 25 \\
\hline 23-09-2017 & $12-10-2017$ & 20 & $18-08-2017$ & $16-09-2017$ & 30 \\
\hline 04-10-2017 & $20-10-2017$ & 16 & $26-08-2017$ & 24-09-2017 & 30 \\
\hline $28-10-2017$ & $16-11-2017$ & 20 & $27-08-2017$ & 22-09-2017 & 27 \\
\hline $26-11-2017$ & $13-12-2017$ & 19 & 04-09-2017 & 02-10-2017 & 29 \\
\hline $28-11-2017$ & $16-12-2017$ & 20 & 11-09-2017 & $11-10-2017$ & 31 \\
\hline $02-12-2017$ & $18-12-2017$ & 17 & $23-09-2017$ & $19-10-2017$ & 27 \\
\hline $08-12-2017$ & $21-12-2017$ & 14 & $10-10-2017$ & 08-11-2017 & 30 \\
\hline $14-02-2017$ & $30-12-2017$ & 17 & 21-11-2017 & $23-12-2017$ & 33 \\
\hline
\end{tabular}


Table 4. Bayes Estimates (BEs) of mixture for two components of Transmuted Weibull Distribution (TWD) along with Posterior Risks (PRs) (in with PRs (in parentheses) under Uniform Prior (UP) and Informative Prior (IP).

\begin{tabular}{|c|c|c|c|c|c|c|c|c|c|c|c|c|c|c|}
\hline \multirow[b]{3}{*}{ LF } & \multicolumn{14}{|c|}{$20 \%$ censoring } \\
\hline & \multicolumn{7}{|c|}{ UP } & \multicolumn{7}{|c|}{ IP } \\
\hline & $\alpha_{1}=2.5$ & $\beta_{1}=1.6$ & $\lambda_{1}=0.3$ & $p_{1}=0.5$ & $\alpha_{2}=3.0$ & $\beta_{2}=1.3$ & $\lambda_{2}=0.4$ & $\alpha_{1}=2.5$ & $\beta_{1}=1.6$ & $\lambda_{1}=0.3$ & $p_{1}=0.5$ & $\alpha_{2}=3.0$ & $\beta_{2}=1.3$ & $\lambda_{2}=0.4$ \\
\hline \multirow{2}{*}{ SELF } & 2.4705 & 1.5832 & 0.3384 & 0.4829 & 3.0773 & 1.2740 & 0.3820 & 2.4956 & 1.6223 & 0.3024 & 0.4827 & 3.0216 & 1.2907 & 0.4136 \\
\hline & $(0.0032)$ & $(0.0927)$ & $(0.0384)$ & $(0.0036)$ & $(0.0016)$ & $(0.0151)$ & $(0.0405)$ & $(0.0034)$ & $(0.0893)$ & $(0.0174)$ & $(0.0037)$ & $(0.0049)$ & $(0.2454)$ & $(0.0201)$ \\
\hline \multirow{2}{*}{ PLF } & 2.4748 & 1.5944 & 0.2910 & 0.4756 & 3.0818 & 1.2799 & 0.4055 & 2.4801 & 1.6177 & 0.3483 & 0.4754 & 3.0290 & 1.3258 & 0.4357 \\
\hline & $(0.0086)$ & $(0.0525)$ & $(0.1052)$ & $(0.0054)$ & $(0.0089)$ & $(0.0118)$ & $(0.1070)$ & $(0.0090)$ & $(0.0509)$ & $(0.0518)$ & $(0.0055)$ & $(0.0149)$ & $(0.1296)$ & $(0.0442)$ \\
\hline \multirow{4}{*}{ QLF } & 2.4534 & 1.6289 & 0.2992 & 0.4815 & 3.0594 & 1.2533 & 0.3957 & 2.4891 & 1.6059 & 0.3263 & 0.4812 & 3.0927 & 1.3091 & 0.4459 \\
\hline & $(0.0235)$ & $(0.0229)$ & $(0.1087)$ & $(0.0087)$ & $(0.0533)$ & $(0.0078)$ & $(0.1093)$ & $(0.0228)$ & $(0.0218)$ & $(0.0886)$ & $(0.0850)$ & $(0.0493)$ & $(0.0401)$ & $(0.0572)$ \\
\hline & \multicolumn{14}{|c|}{$40 \%$ censoring } \\
\hline & \multicolumn{7}{|c|}{ UP } & \multicolumn{7}{|c|}{ IP } \\
\hline $\mathbf{L F}$ & $\alpha_{1}=2.5$ & $\beta_{1}=1.6$ & $\lambda_{1}=0.3$ & $p_{1}=0.5$ & $\alpha_{2}=3.0$ & $\beta_{2}=1.3$ & $\lambda_{2}=0.4$ & $\alpha_{1}=2.5$ & $\beta_{1}=1.6$ & $\lambda_{1}=0.3$ & $p_{1}=0.5$ & $\alpha_{2}=3.0$ & $\beta_{2}=1.3$ & $\lambda_{2}=0.4$ \\
\hline \multirow{2}{*}{ SELF } & 2.4658 & 1.5938 & 0.2947 & 0.5212 & 3.0780 & 1.3103 & 0.4022 & 2.4932 & 1.6006 & 0.2968 & 0.5166 & 3.0468 & 1.3220 & 0.4146 \\
\hline & $(0.0038)$ & $(0.1138)$ & $(0.0486)$ & $(0.0043)$ & $(0.0009)$ & $(0.0164)$ & $(0.0496)$ & $(0.0042)$ & $(0.0992)$ & $(0.0180)$ & $(0.0056)$ & $(0.0119)$ & $(0.2576)$ & $(0.0204)$ \\
\hline \multirow{2}{*}{ PLF } & 2.4702 & 1.6253 & 0.3397 & 0.5226 & 3.0860 & 1.3155 & 0.4277 & 2.4774 & 1.6279 & 0.3202 & 0.5182 & 3.0636 & 1.3259 & 0.4220 \\
\hline & $(0.0092)$ & $(0.0629)$ & $(0.1201)$ & $(0.0098)$ & $(0.0112)$ & $(0.0123)$ & $(0.1190)$ & $(0.0104)$ & $(0.0547)$ & $(0.0648)$ & $(0.0062)$ & $(0.0334)$ & $(0.1357)$ & $(0.0447)$ \\
\hline \multirow{2}{*}{ QLF } & 2.4799 & 1.6379 & 0.2891 & 0.5150 & 3.0463 & 1.2922 & 0.4227 & 2.4561 & 1.6106 & 0.3066 & 0.5010 & 3.0816 & 1.3106 & 0.4484 \\
\hline & $(0.0217)$ & $(0.0249)$ & $(0.1184)$ & $(0.0039)$ & $(0.0667)$ & $(0.0082)$ & $(0.1234)$ & $(0.0234)$ & $(0.0245)$ & $(0.916)$ & $(0.0041)$ & $(0.1021)$ & $(0.0423)$ & $(0.1182)$ \\
\hline
\end{tabular}

Table 5. 95\% Bayesian credible intervals of mixture of the two components of Transmuted Weibull Distribution (TWD) using Uniform Prior (UP) and Informative Prior (IP).

\begin{tabular}{cccccc}
\hline \multirow{2}{*}{ Censoring rate } & Parameters & \multicolumn{2}{c}{ UP } & \multicolumn{2}{c}{ IP } \\
\cline { 2 - 6 } & & Lower limit & Upper limit & Lower limit & Upper limit \\
\hline$\alpha_{1}=2.5$ & 0.0587 & 2.8914 & 0.0695 & 2.8940 \\
$\alpha_{2}=3.0$ & 0.0286 & 3.7778 & 0.0599 & 3.0195 \\
$\beta_{1}=1.6$ & 0.2986 & 1.7778 & 0.3563 & 1.6008 \\
$20 \%$ & $\beta_{2}=1.3$ & 0.2184 & 1.6721 & 0.3549 & 1.8350 \\
$\lambda_{1}=0.3$ & 0.1696 & 0.6763 & 0.2144 & 0.5770 \\
$\lambda_{2}=0.4$ & 0.1797 & 0.6833 & 0.3210 & 0.6832 \\
$p_{1}=0.5$ & 0.1125 & 3.3063 & 0.1304 & 3.5657 \\
& & & & \\
& $\alpha_{1}=2.5$ & 0.0655 & 2.7424 & 0.1035 & 3.6075 \\
$\alpha_{2}=3.0$ & 0.0338 & 4.5974 & 0.0945 & 3.9551 \\
$\beta_{1}=1.6$ & 0.3038 & 2.1947 & 0.3697 & 1.6238 \\
$\beta_{2}=1.3$ & 0.2290 & 3.7483 & 0.3920 & 1.9520 \\
$\lambda_{1}=0.3$ & 0.1774 & 0.6814 & 0.2158 & 0.5875 \\
$\lambda_{2}=0.4$ & 0.1832 & 0.6875 & 0.3227 & 0.6857 \\
$p_{1}=0.5$ & 0.1289 & 4.9770 & 0.1455 & 4.5908 \\
\hline
\end{tabular}

\section{Acknowledgement}

The authors are thankful to the anonymous reviewers and the corresponding editor for the constructive comments that have helped to improve the article.

\section{References}

1. Shaw, W. and Buckley, I., The Alchemy of Probability Distributions: Beyond Gram-Charlier Expansions and a Skew-kurtotic-Normal Distribution from a Rank 
Transmutation Map (Research Report). King's College, London, U.K (2007).

2. Bhati, D., Kumawat, P., and Gómez- Dèniz, E. "A new count model generated from mixed poison transmuted exponential family with an application to health care data", arXiv: 1504.01097v2 (2016).

3. Shaw, W.T. and Buckley, I.R.C. "The alchemy of probability distributions: Beyond gram-charlier expansions and a skew-kurtotic normal distribution from a rank transmutation map", ArXiv Preprint: 0901.0434v1 [qfin.ST] 5 Jan 2009 (2009).

4. Jandhyala, V.K., Fotopoulos, S.B., and Evaggelopoulos, N. "Change-point methods for weibull models with applications to detection of trends in extreme temperatures", Environmetrics, 10, pp. 547-564 (1999).

5. Khan, M.S., King, R., and Hudson, I.L. "Transmuted new generalized weibull distribution for lifetime modeling", Communications for Statistical Applications and Methods, 23(5), pp. 363-383 (2016).

6. Mudholkar, G., Srivastava, D., and Kollia, G. "A generalization of the weibull distribution with application to the analysis of survival data", Journal of the American Statistical Association, 91(436), pp. 15751583 (1996).

7. Aryal, G.R. and Tsokos, C.P. "Transmuted weibull distribution: A generalization of the weibull probability distribution", European Journal of Pure and Applied Mathematics, 4(2), pp. 89-102 (2011).

8. Khan, M.S. and King, R. "Transmuted modified weibull distribution: A generalization of the modified weibull probability distribution", European Journal of Pure and Applied Mathematics, 6(1), pp. 66-88 (2013).

9. Merovci, F., Elbatal, I., and Ahmed, A. "The transmuted generalized inverse weibull distribution", Austrian Journal of Statistics, 43(2), pp. 119-131 (2014).

10. Abdurrahman, S.A. "Comparing different estimators of three parameters for transmuted weibull distribution", Global Journal of Pure and Applied Mathematics, 13(9), pp. 5115-5128 (2017).

11. Nofal, Z.M. and El Gebaly, Y.M. "The generalized transmuted weibull distribution for lifetime data", Pakistan Journal of Statistics and Operation Research, 8(2), pp. 355-378 (2017).

12. Newcomb, S. "A generalized theory of the combination of observations so as to obtain best result", American Journal of Mathematics, 8, pp. 343-366 (1886).

13. Feroze, N. and Aslam, M. "Bayesian analysis of doubly censored lifetime data using two-component mixture of weibull distribution", Journal of National Science Foundation of Sri Lanka, 42(4), pp. 325-334 (2014).

14. Sindhu, T.N., Feroze, N., and Aslam, M. "Doubly censored data from two - component mixture of inverse weibull distributions: Theory and applications", Journal of Modern Applied Statistical Methods, 15(2), pp. 322-349 (2016).
15. Aslam, M., Tahir, M., Hussain, Z., and Al-Zahrani, B. "A 3-component mixture of Rayleigh distributions: Properties and estimation in Bayesian framework", Public Library of Science One, pp. 1-14 (2015).

16. Aslam, M., Tahir, M., and Hussain, Z. "Reliability analysis of three-component mixture of distributions", Scientia Iranica, Transactions E: Industrial Engineering, 25, pp. 1768-1781 (2018).

17. Tahir, M., Aslam, M., Hussain. H., Abid, M., and Bhatti, S.H. "Bayesian analysis of heterogeneous doubly censored lifetime data using the 3-component mixture of Rayleigh distributions: A Monte Carlo simulation study", Scientia Iranic, 26(3), pp. 17891808 (2019).

18. Ateya, S.F. "Maximum likelihood estimation under a finite mixture of generalized exponential distributions based on censored data", Statistical Papers, 55(2), pp. 311-325 (2014).

19. Benaicha, H. and Chaker, A. "Weibull mixture modelfor reliability analysis", International Review of Electrical Engineering, 9(5), pp. 986-990 (2014).

20. Kalbfleisch, J.D. and Prentice, R.L., The Statistical Analysis of Failure Time Data, Wiley, New York (1980).

21. Gill, R.D. Censoring and Stochastic Integrals, Mathematical Centre Tracts 124, Mathematisch Centrum, Amsterdam (1980).

22. Miller, R.G. Jr, Survival Analysis, John Wiley and Sons, Inc., New York, pp. 104-118 (1981).

23. Mendenhall, W. and Hader, R.A. "Estimation of parameters of mixed exponentially distributed failure time distributions from censored life test data", Biometrika, 45(3-4), pp. 504-520 (1958).

24. Lindley, D.V. "Theory and practice of Bayesian statistics", The Statistician, 32, pp. 1-11 (1983).

25. Walters, C. and Ludwig, D. "Calculation of bayes posterior probability distributions for key population parameters: a specified approach", Canadian Journal of Fisheries and Aquatic Sciences, 51(3), pp. 713-722 (1994).

26. Laplace, P.S., Theorie Analytique des Probabilities, Veuve.Courcier, Paris (1812).

27. Punt, A.E. and Butterworth, D.S. "Why do Bayesian and maximum likelihood assessments of the beringchukchi-beaufort seas stock of bowhead whales differ?", Journal of Cetacean Research and Management, 2(2), pp. 125-133 (2000).

28. Punt, A.E. and Walker, T.I. "Stock assessment and risk analysis for the school shark galeorhinus galeus (Linnaeus) off southern Australia", Marina and Freshwater Research, 49, pp. 719-731 (1998).

29. Abid, M., Naeem, A., Hussain, Z., Riaz, M., and Tahir, M. "Investigating the impact of simple and mixture priors on estimating sensitive proportion through a general class of randomized response models", Scientia Iranica, 26(2), pp. 1009-1022 (2019). 
30. Franz, J. "Posterior distribution and loss functions for parameter estimation in weibull processes", Economic Quality Control, 21(1), pp. 31-42 (2006).

31. Ali, S., Aslam, M., Nasir, A., and Kazmi, S.M.A. "Scale parameter estimation of the Laplace model using different asymmetric loss functions", International Journal of Statistics and Probability, 1(1), pp. 105-127 (2012).

32. Gauss, C.F., Least Squares Method for the Combinations of Observations, Translated by J. Bertrand 1955, Mallet-Bachelier, Paris (1810).

33. Legendre, A.M., Nouvelles Methods pour la Determination des Orbites des Cometes, F. Didot (1805).

34. Norstrom, J.G. "The use of precautionary loss functions in risk analysis", IEEE Transactions on Reliability, 45(1), pp. 400-403 (1996).

35. Ali, S. "Mixture of the inverse Rayleigh distribution: properties and estimation in a Bayesian framework", Applied Mathematical Modelling, 39, pp. 515-530 (2015).

36. Ali, S. "On the Bayesian estimation of the weighted lindley distribution", Journal of Statistical Computation and Simulation, 85(5), pp. 855-880 (2015).

37. Casella, G. and George, E.I. "Explaining the gibbs sampler", American. Statistician, 46(3), pp. 167-174 (1992).

38. Metropolis, N. and Ulam, S. "The Monte Carlo method", Journal of the American Statistical Association, 44, pp. 335-341 (1949).

39. Metropolis, N., Rosenbluth, A., Rosenbluth, M., Teller, A., and Teller, E. "Equations of state calculation by fast computing machines", Journal of Chemical Physics, 21(6), pp. 1087-1092 (1953).

40. R Core Team R: A Language and Environment for Statistical Computing. $R$ Foundation for Statistical Computing, Vienna, Austria.URL http://www.Rproject.org/ (2013).

41. Charnigo, R., Francoeur, M., Kenkel, P., Mengüc, M.P., Hall, B., and Srinivasan, C. "Credible intervals for nanoparticle charactiristics", Journal of Quantitative Spectroscopy \& Radiative Transfer, 113(2), pp. 182-193 (2012).

42. Eberlya, L.E. and Casella, G. "Estimating Bayesian credible intervals", Journal of Statistical Planning and Inference, 112, pp. 115-132 (2003).

43. Dasgupta, R. "On the distribution of Burr with applications", Sankhya B, 73, pp. 1-19 (2011).
44. Bakouch, H., Jamal, F., Chesneau, C., and Nasir, A. "A new transmuted family of distributions: Properties and estimation with applications", https://hal.archives-ouvertes.fr/hal-01570370v3

(2017).

\section{Appendix A}

For $f(x ; \Omega)=p_{1} f_{1}\left(x ; \Omega_{1}\right)+p_{2} f_{2}\left(x ; \Omega_{2}\right)$ and $p_{1}+p_{2} \leq$ 1 , some algebraic manipulations yields the following likelihood form:

$$
\begin{aligned}
& L(x ; \Omega) \propto p_{1}^{r_{1}+1-1}\left(1-p_{1}\right)^{r_{2}+1-1} \alpha_{1}^{r_{1}+1-1} \\
& \exp \left\{-\alpha_{1} \sum_{j=1}^{r_{1}} \log \left(\frac{1}{x_{1 j}}\right)\right\} \alpha_{2}^{r_{2}+1-1} \\
& \exp \left\{-\alpha_{2} \sum_{j=1}^{r_{2}} \log \left(\frac{1}{x_{2 j}}\right)\right\} \frac{1}{\left(\beta_{1}\right)^{r_{1}-1+1}} \\
& \exp \left(-\frac{\sum_{j=1}^{r_{1}} x_{1 j}^{\alpha_{2}}}{\beta_{1}}\right) \frac{1}{\left(\beta_{2}\right)^{r_{2}-1+1}} \\
& \exp \left(-\frac{\sum_{j=1}^{r_{1}} x_{2 j}^{\alpha_{2}}}{\beta_{2}}\right) \exp \left[\sum _ { j = i } ^ { r _ { 1 } } \operatorname { l o g } \left\{1-\lambda_{1}+2 \lambda_{1}\right.\right. \\
& \left.\left.\exp \left(-\frac{x_{1 j}^{\alpha_{1}}}{\beta_{1}}\right)\right\}\right] \exp \left[\sum _ { j = i } ^ { r _ { 2 } } \operatorname { l o g } \left\{1-\lambda_{2}+2 \lambda_{2}\right.\right. \\
& \left.\left.\exp \left(-\frac{x_{2 j}^{\alpha_{2}}}{\beta_{2}}\right)\right\}\right]\left[1-p_{1} \exp \left(-\frac{T^{\alpha_{1}}}{\beta_{1}}\right)\right. \\
& \left\{1-\lambda_{1}+2 \lambda_{1} \exp \left(-\frac{T^{\alpha_{1}}}{\beta_{1}}\right)\right\}-\left(1-p_{1}\right) \\
& \left.\exp \left(-\frac{T^{\alpha_{2}}}{\beta_{2}}\right)\left\{1-\lambda_{2}+\lambda_{2} \exp \left(-\frac{T^{\alpha_{2}}}{\beta_{2}}\right)\right\}\right]^{n-r}
\end{aligned}
$$

Figure A.1 shows graphs of the marginal posterior densities of parameters $\left(\alpha_{1}, \alpha_{2}, \beta_{1}, \beta_{2}, \lambda_{1} \lambda_{2}, p_{1}\right)$ assuming the UP and the $1 \mathrm{P}$ and the hepatitis data. From Figure A.1a and A.1b and A.1e \& A.1f, it is noted that the plots of marginal posterior densities for the scale and the shape component for the scale and the shape parameters $\left(\alpha_{2}, \beta_{2}\right)$ tend to be more peaked for $20 \%$ censoring than for the $40 \%$. This is because more information was lost in the case of a high censoring rate (Figure A.1c and A.1d \& A.1g and A.1h). Also, the graphs of marginal posterior densities for transmuted parameters $\left(\lambda_{1}, \lambda_{2}\right)$ are also symmetrical, as shown in Figures A.1i to A.1l, and the same is true for the 


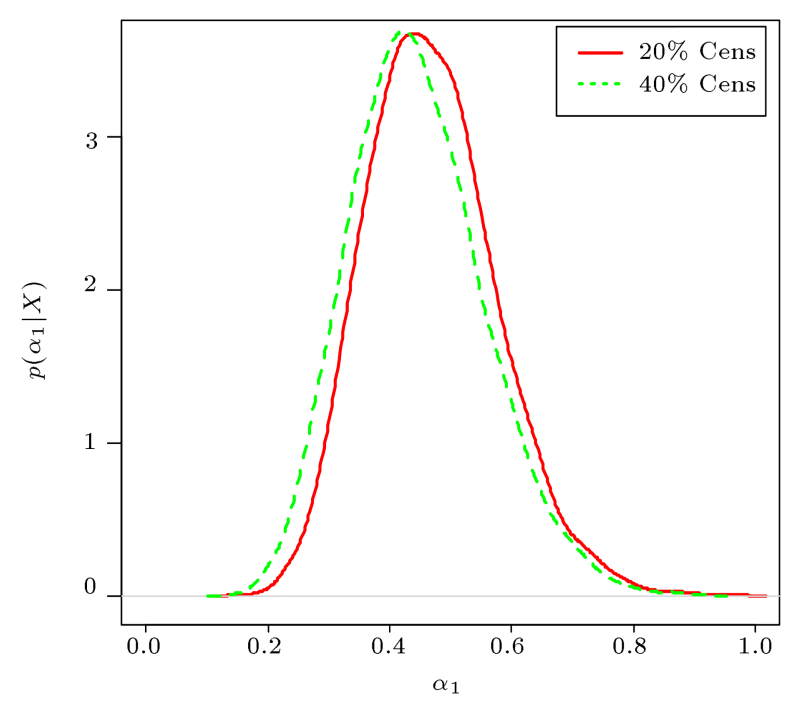

Figure A.1a. Posterior density of $\alpha_{1}$ using Uniform Prior (UP).

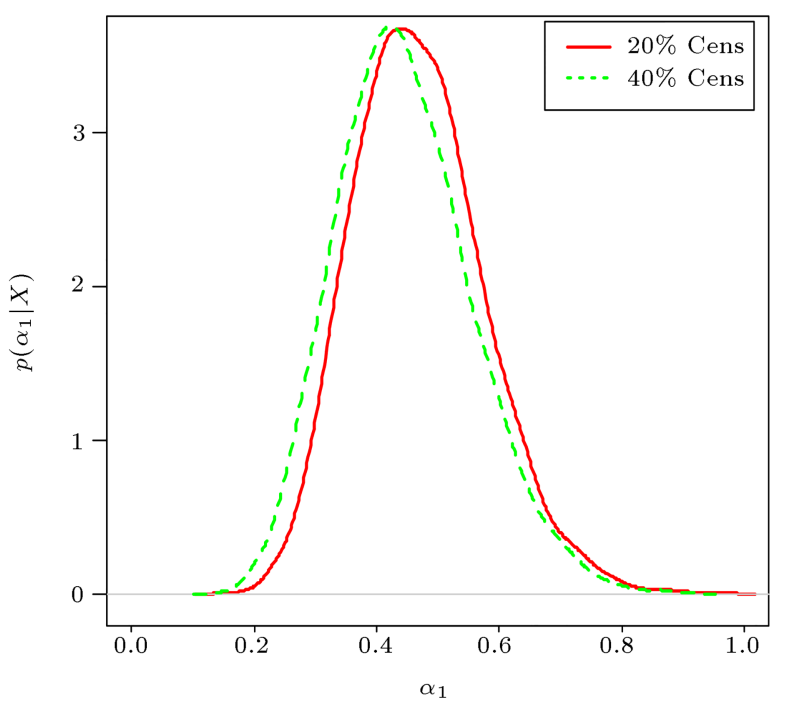

Figure A.1b. Posterior density of $\alpha_{1}$ using Informative Prior (IP).

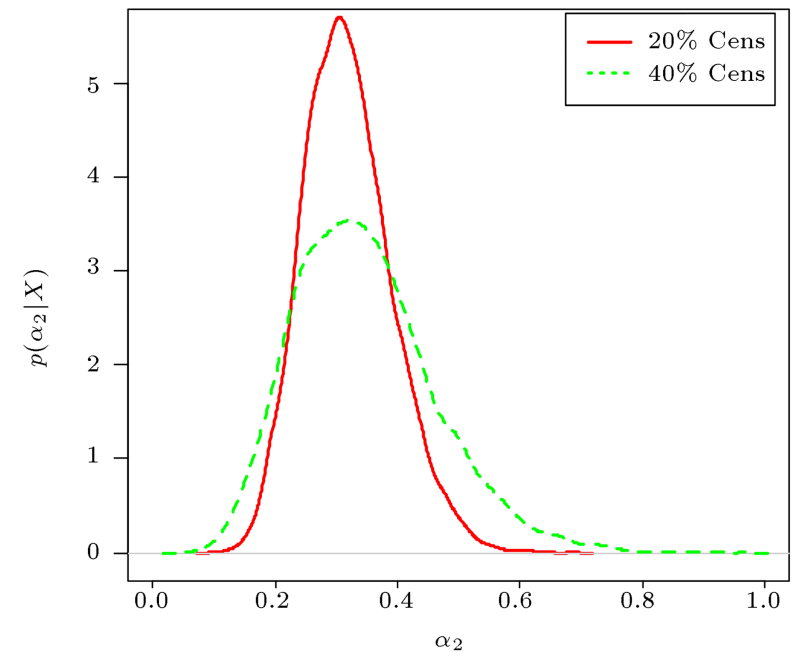

Figure A.1c. Posterior density of $\alpha_{2}$ using Uniform Prior (UP).

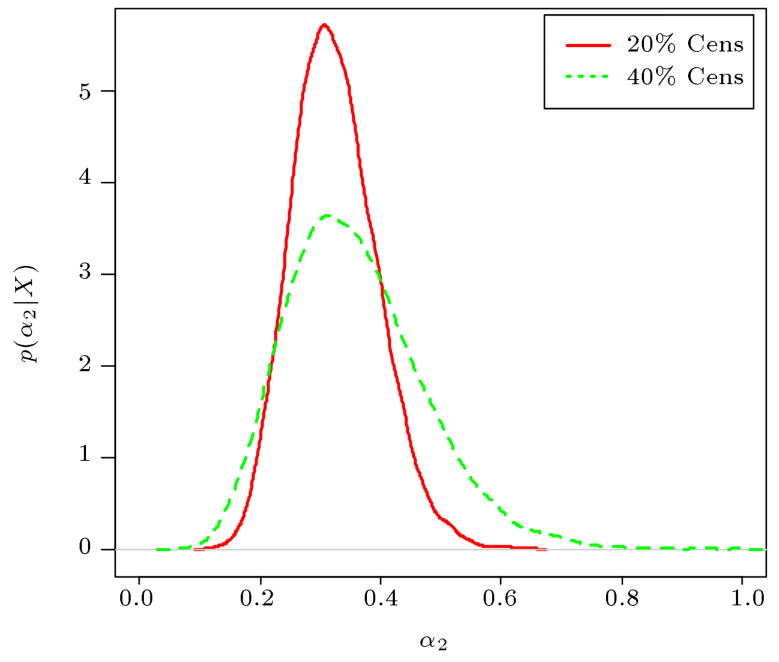

Figure A.1d. Posterior density of $\alpha_{2}$ using Informative Prior (IP).

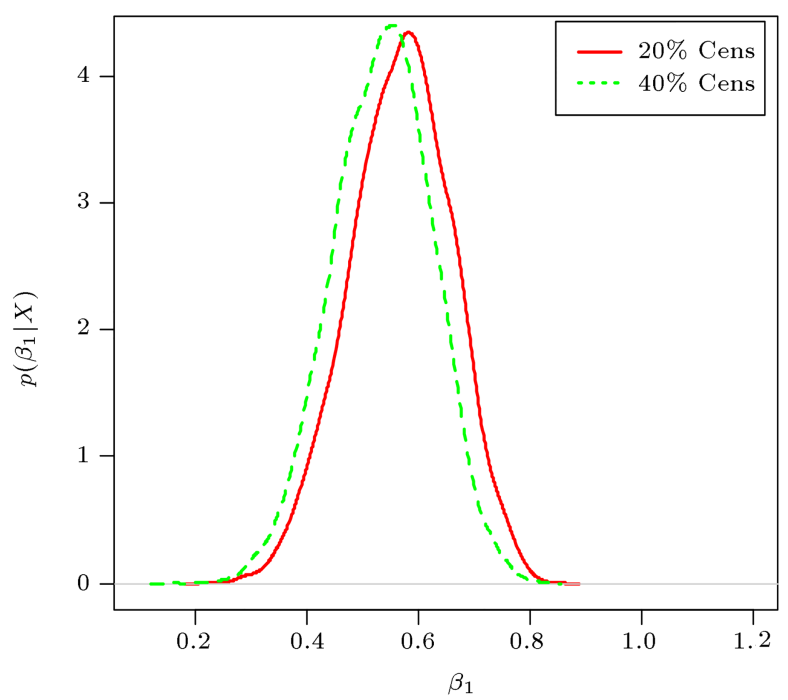

Figure A.1e. Posterior density of $\beta_{1}$ using Uniform Prior (UP).

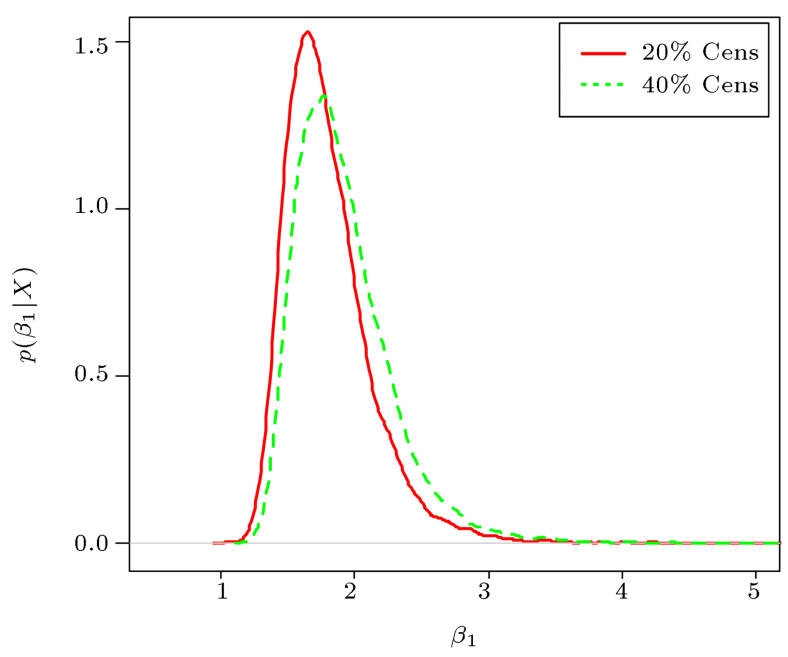

Figure A.1f. Posterior density of $\beta_{1}$ using Informative Prior (IP). 


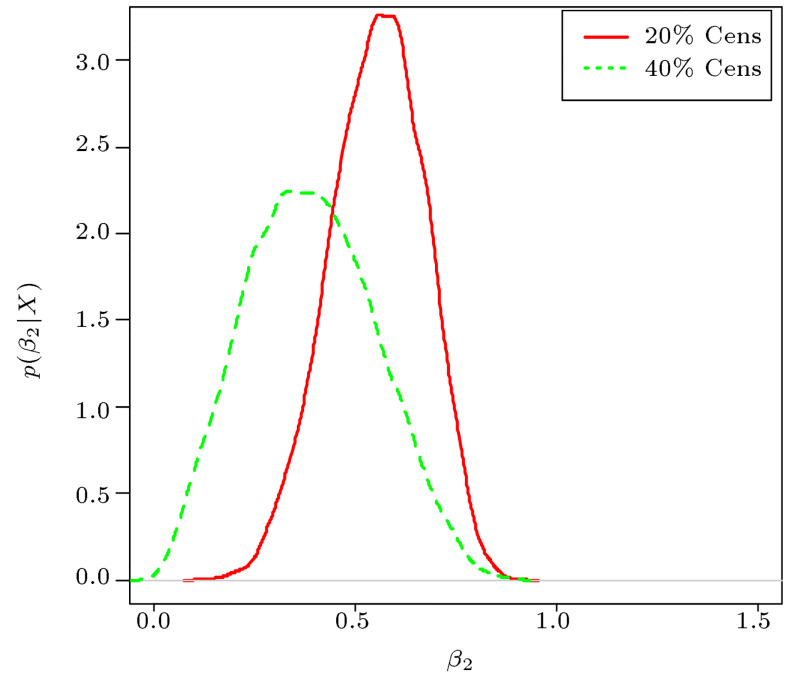

Figure A.1g. Posterior density of $\beta_{2}$ using Uniform Prior (UP).

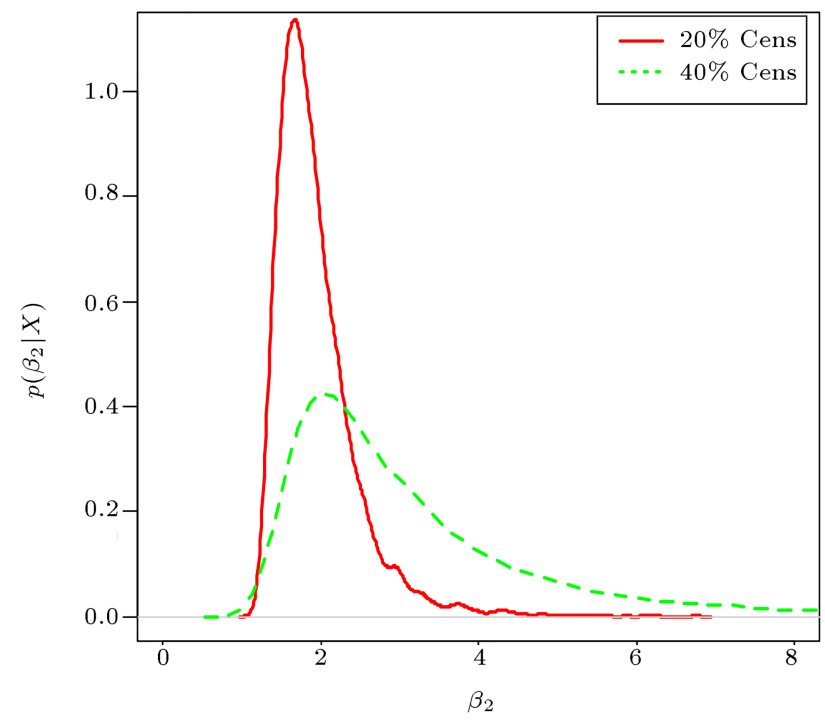

Figure A.1h. Posterior density of $\beta_{2}$ using Informative Prior (IP).

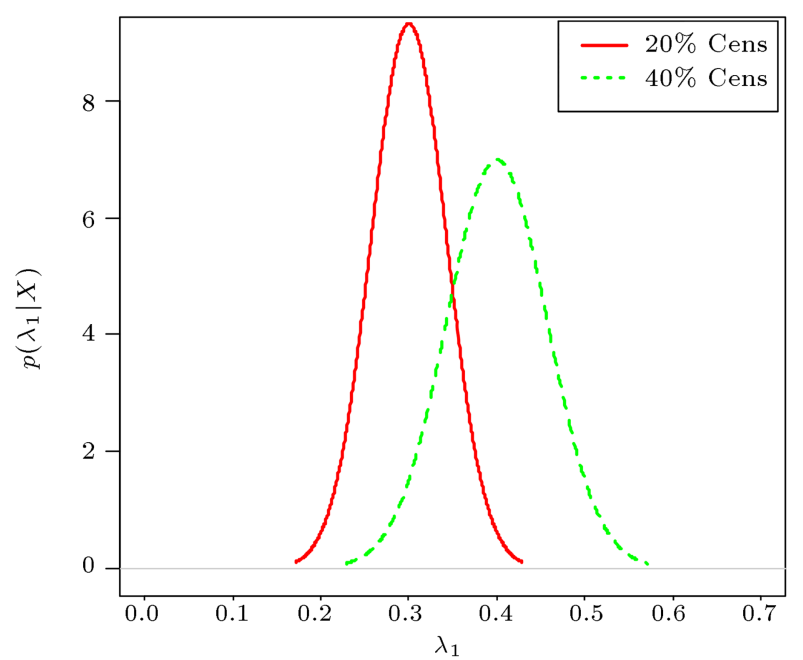

Figure A.1i. Posterior density of $\lambda_{1}$ using Uniform Prior (UP).

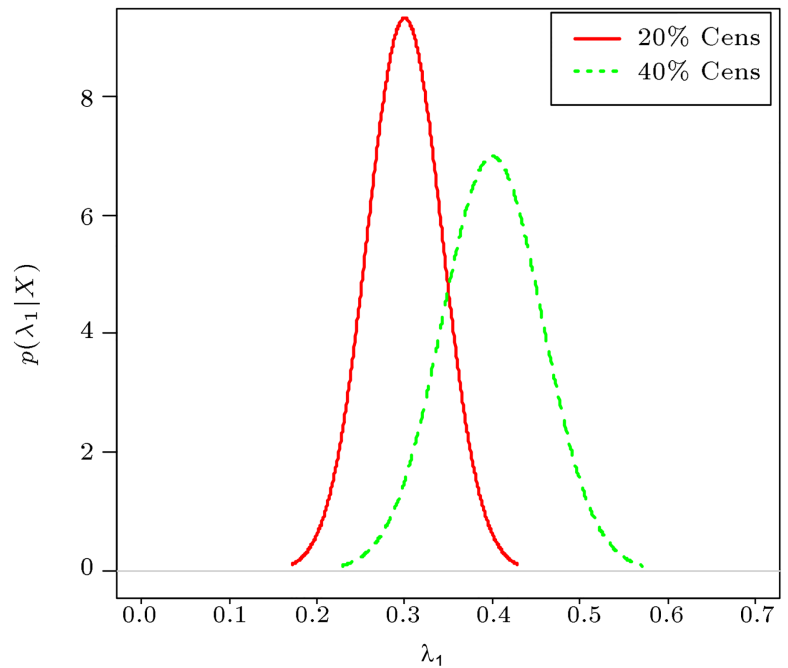

Figure A.1j. Posterior density of $\lambda_{1}$ using Informative Prior (IP).

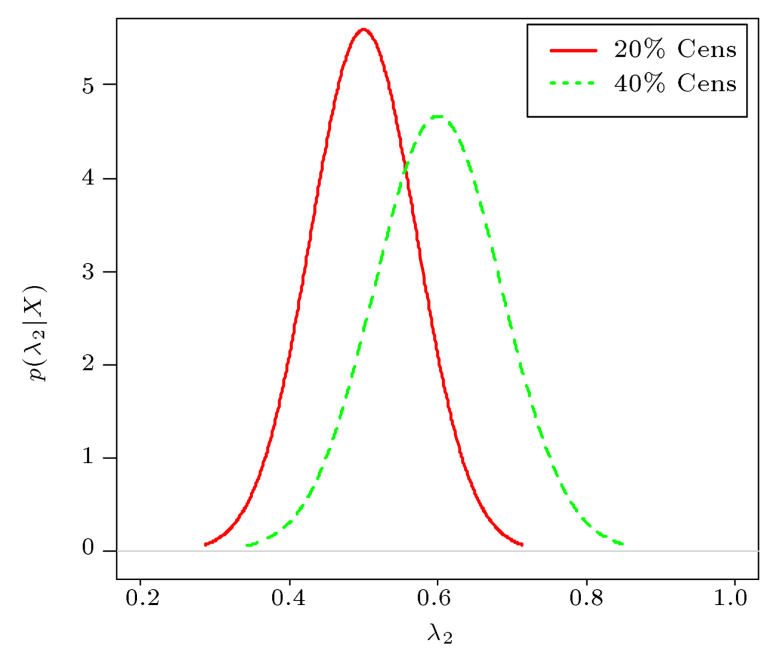

Figure A.1k. Posterior density of $\lambda_{2}$ using Uniform Prior (UP).

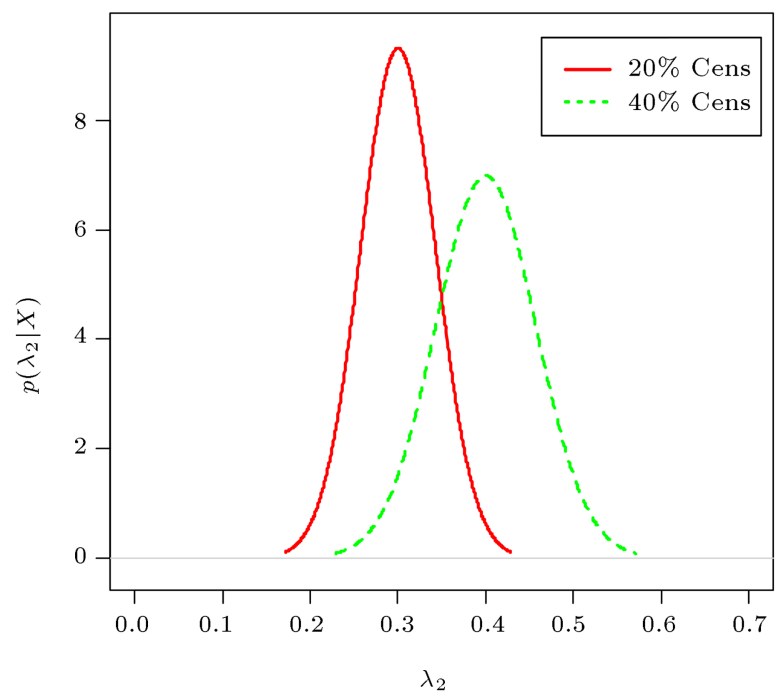

Figure A.11. Posterior density of $\lambda_{2}$ using Informative Prior (IP). 


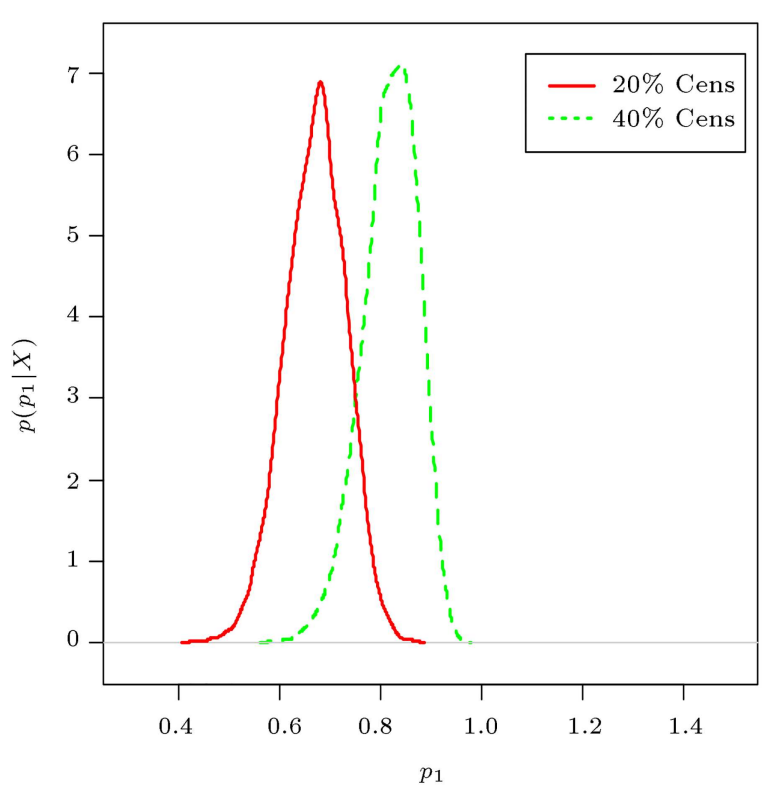

Figure A.1m. Posterior density of $p_{1}$ using Uniform Prior (UP).

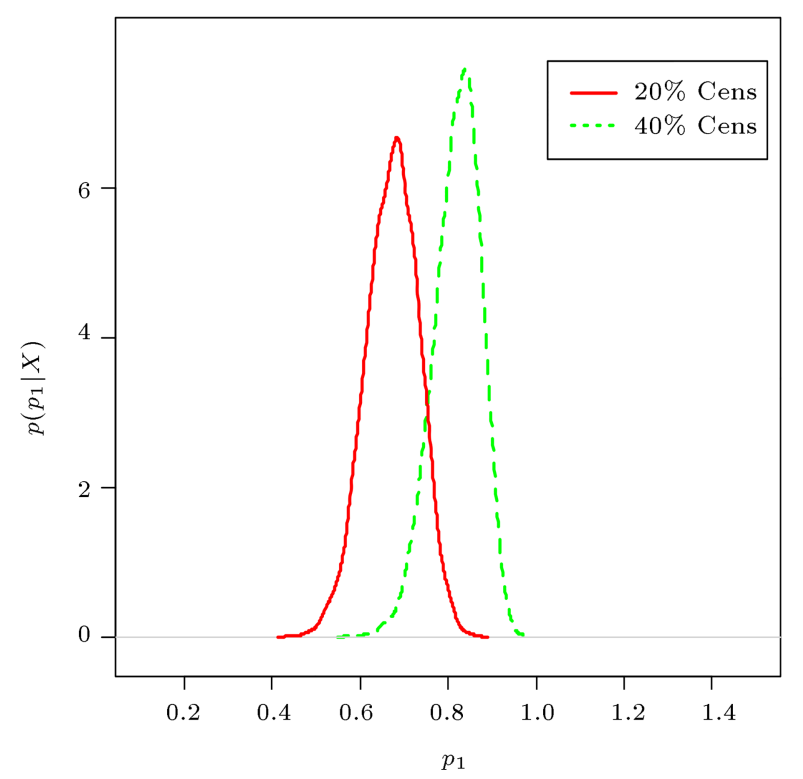

Figure A.1n. Posterior density of $p_{1}$ using Informative Prior (IP).

posterior density of the mixing weight (Figures A.1m and A.1n).

\section{Real data set-II}

The second data set consists of 50 observations (in millimeters), the hole diameter is $12 \mathrm{~mm}$ and the sheet thickness is $3.15 \mathrm{~mm}$, as reported by Dasgupta [43]. The data are: $0.04,0.02,0.06,0.12,0.14,0.08,0.22$, $0.12,0.08,0.26,0.24,0.04,0.14,0.16,0.08,0.26,0.32$, $0.28,0.14,0.16,0.24,0.22,0.12,0.18,0.24,0.32,0.16$, $0.14,0.08,0.16,0.24,0.16,0.32,0.18,0.24,0.22,0.16$,
$0.12,0.24,0.06,0.02,0.18,0.22,0.14,0.06,0.04$, $0.14,0.26,0.18,0.16$. Dasgupta analyzed the Burr distribution by using the same data set and compared the process stability by bootstrapping the distribution of coefficient of variation under an extreme value model. He concludes that an extreme value distribution may explain the data well. Recently, Bakouch et al. [44] used this data set to evaluate the performance of the Transmuted General (TG) family of distributions by fitting four sub-models, namely; Transmuted Burr (TB) distribution, Transmuted Weibull (TW) distribution, Transmuted Gamma (TGa) distribution, and Transmuted Gompterz ( $\mathrm{TGz}$ ) distribution. In order to perform the Bayesian analysis assuming the twocomponent mixture of TWD, the data was randomly assembled into two subpopulations using probabilistic mixing weight $p_{1}=0.5$ and $T=0.24$. The summary of results are as follows:

$$
\begin{aligned}
& n_{1}=25, \quad r_{1}=18, \quad p_{1}=0.5 \\
& \sum_{j=1}^{r_{1}} x_{1 j}=2.22 \\
& \sum_{j=1}^{r_{1}} \log x_{1 j}=-40.51 \\
& n_{2}=25, \quad r_{2}=19 \\
& \sum_{j=1}^{r_{2}} x_{2 j}=2.48 \\
& \sum_{j=1}^{r_{2}} \log x_{2 j}=-41.53 .
\end{aligned}
$$

The goodness of fit for the distribution is tested by using the $\chi^{2}$-statistic against the Weibull and transmuted Weibull distributions and the obtained Pvalue is 0.2290 at the $5 \%$ level of significance. Thus, it is safe to conclude that the proposed distribution provides the best fit for the data set. The two-component mixture of TWD was applied to the data set given in Table A.5 by adopting the methodology discussed previously. More specifically, the Bayesian analysis was performed to estimate the unknown parameters assuming UP and IP under SELF, PLF and QLF. The results of the BEs, the PRs (in parenthesis) and interval estimates for the parameters have been summarized in tables intervals (Tables A.6 and A.7). The BEs and the credible intervals (Tables A.6 and A.7) based on IP are more efficient than the UP prior. For both priors, the SELF provided more efficient estimates for transmuted parameters $\left(\lambda_{1}, \lambda_{2}\right)$ and proportion parameter $\left(p_{1}\right)$. 
Table A.1. Bayes Estimates (BEs) of mixture for two components of Transmuted Weibull Distribution (TWD) along with Posterior Risks (PRs) (in parentheses) under Uniform Prior (UP) and Informative Prior (IP).

\begin{tabular}{|c|c|c|c|c|c|c|c|c|c|c|c|c|c|c|c|}
\hline \multirow[b]{3}{*}{$\mathbf{L F}$} & \multicolumn{15}{|c|}{$20 \%$ censoring } \\
\hline & \multicolumn{8}{|c|}{ UP } & \multicolumn{7}{|c|}{ IP } \\
\hline & $n$ & $\alpha_{1}=2.5$ & $\beta_{1}=1.6$ & $\lambda_{1}=0.3$ & $p_{1}=0.5$ & $\alpha_{2}=3.0$ & $\beta_{2}=1.3$ & $\lambda_{2}=0.4$ & $\alpha_{1}=2.5$ & $\beta_{1}=1.6$ & $\lambda_{1}=0.3$ & $p_{1}=0.5$ & $\alpha_{2}=3.0$ & $\beta_{2}=1.3$ & $\lambda_{2}=0.4$ \\
\hline \multirow{4}{*}{ SELF } & 20 & $\begin{array}{c}1.8925 \\
(0.2593)\end{array}$ & $\begin{array}{c}1.5085 \\
(0.1023)\end{array}$ & $\begin{array}{c}0.3769 \\
(0.0339)\end{array}$ & $\begin{array}{c}0.3894 \\
(0.0124)\end{array}$ & $\begin{array}{c}2.5369 \\
(0.2827)\end{array}$ & $\begin{array}{c}1.4061 \\
(0.0306)\end{array}$ & $\begin{array}{c}0.4595 \\
(0.0442)\end{array}$ & $\begin{array}{c}2.7200 \\
(0.2309)\end{array}$ & $\begin{array}{c}1.4753 \\
(0.2420)\end{array}$ & $\begin{array}{c}0.3621 \\
(0.0052)\end{array}$ & $\begin{array}{c}0.4479 \\
(0.0116)\end{array}$ & $\begin{array}{c}3.4721 \\
(0.2860)\end{array}$ & $\begin{array}{c}1.3678 \\
(0.1676)\end{array}$ & $\begin{array}{c}0.4669 \\
(0.0338)\end{array}$ \\
\hline & 40 & $\begin{array}{c}2.1738 \\
(0.1817)\end{array}$ & $\begin{array}{c}1.5511 \\
(0.0248)\end{array}$ & $\begin{array}{c}0.3480 \\
(0.0309)\end{array}$ & $\begin{array}{c}0.4408 \\
(0.0070)\end{array}$ & $\begin{array}{c}2.8638 \\
(0.1358)\end{array}$ & $\begin{array}{c}1.3640 \\
(0.0082)\end{array}$ & $\begin{array}{c}0.4464 \\
(0.0396)\end{array}$ & $\begin{array}{c}2.5753 \\
(0.2002)\end{array}$ & $\begin{array}{c}1.5673 \\
(0.2195)\end{array}$ & $\begin{array}{c}0.3287 \\
(0.0009)\end{array}$ & $\begin{array}{c}0.4443 \\
(0.0069)\end{array}$ & $\begin{array}{c}3.0435 \\
(0.1649)\end{array}$ & $\begin{array}{c}1.3837 \\
(0.1372)\end{array}$ & $\begin{array}{c}0.4291 \\
(0.0277)\end{array}$ \\
\hline & 60 & $\begin{array}{c}2.3918 \\
(0.1094)\end{array}$ & $\begin{array}{c}1.5815 \\
(0.0102)\end{array}$ & $\begin{array}{c}0.3412 \\
(0.0306)\end{array}$ & $\begin{array}{c}0.4581 \\
(0.0047)\end{array}$ & $\begin{array}{c}3.0648 \\
(0.1026)\end{array}$ & $\begin{array}{c}1.3499 \\
(0.0047)\end{array}$ & $\begin{array}{c}0.4402 \\
(0.0392)\end{array}$ & $\begin{array}{c}2.5371 \\
(0.1136)\end{array}$ & $\begin{array}{c}1.5871 \\
(0.1280)\end{array}$ & $\begin{array}{c}0.3262 \\
(0.0007)\end{array}$ & $\begin{array}{c}0.4792 \\
(0.0047)\end{array}$ & $\begin{array}{l}3.0657 \\
(0.1084)\end{array}$ & $\begin{array}{l}1.3491 \\
(0.1048)\end{array}$ & $\begin{array}{c}0.4161 \\
(0.0205)\end{array}$ \\
\hline & 100 & $\begin{array}{c}2.5919 \\
(0.0948)\end{array}$ & $\begin{array}{c}1.6008 \\
(0.0036)\end{array}$ & $\begin{array}{c}0.3339 \\
(0.0290)\end{array}$ & $\begin{array}{c}0.4999 \\
(0.0030)\end{array}$ & $\begin{array}{c}3.0679 \\
(0.0988)\end{array}$ & $\begin{array}{c}1.3186 \\
(0.0029)\end{array}$ & $\begin{array}{c}0.4305 \\
(0.0378)\end{array}$ & $\begin{array}{c}2.4842 \\
(0.0931)\end{array}$ & $\begin{array}{c}1.6046 \\
(0.0937)\end{array}$ & $\begin{array}{c}0.30000 \\
(0.0002)\end{array}$ & $\begin{array}{c}0.4997 \\
(0.0030)\end{array}$ & $\begin{array}{c}3.0024 \\
(0.0946)\end{array}$ & $\begin{array}{l}1.3125 \\
(0.0827)\end{array}$ & $\begin{array}{c}0.4190 \\
(0.0183)\end{array}$ \\
\hline \multirow{4}{*}{ PLF } & 20 & $\begin{array}{c}2.0225 \\
(0.2602)\end{array}$ & $\begin{array}{c}1.5933 \\
(0.0468)\end{array}$ & $\begin{array}{c}0.4194 \\
(0.1050)\end{array}$ & $\begin{array}{c}0.4051 \\
(0.0313)\end{array}$ & $\begin{array}{c}2.8609 \\
(0.2147)\end{array}$ & $\begin{array}{c}1.4032 \\
(0.0304)\end{array}$ & $\begin{array}{c}0.5053 \\
(0.0915)\end{array}$ & $\begin{array}{c}2.3992 \\
(0.2662)\end{array}$ & $\begin{array}{c}1.4577 \\
(0.2471)\end{array}$ & $\begin{array}{c}0.3591 \\
(0.0053)\end{array}$ & $\begin{array}{c}0.4129 \\
(0.0280)\end{array}$ & $\begin{array}{c}3.3125 \\
(0.2708)\end{array}$ & $\begin{array}{l}1.3847 \\
(0.0160)\end{array}$ & $\begin{array}{c}0.4479 \\
(0.0365)\end{array}$ \\
\hline & 40 & $\begin{array}{c}2.2444 \\
(0.1411)\end{array}$ & $\begin{array}{c}1.6023 \\
(0.0244)\end{array}$ & $\begin{array}{c}0.3899 \\
(0.0937)\end{array}$ & $\begin{array}{c}0.4488 \\
(0.0160)\end{array}$ & $\begin{array}{c}2.9389 \\
(0.1502)\end{array}$ & $\begin{array}{c}1.3983 \\
(0.0087)\end{array}$ & $\begin{array}{c}0.4795 \\
(0.0863)\end{array}$ & $\begin{array}{c}2.6519 \\
(0.1531)\end{array}$ & $\begin{array}{c}1.5748 \\
(0.1683)\end{array}$ & $\begin{array}{c}0.3301 \\
(0.0027)\end{array}$ & $\begin{array}{c}0.4520 \\
(0.0153)\end{array}$ & $\begin{array}{c}3.1490 \\
(0.1748)\end{array}$ & $\begin{array}{c}1.3420 \\
(0.0072)\end{array}$ & $\begin{array}{c}0.4201 \\
(0.0318)\end{array}$ \\
\hline & 60 & $\begin{array}{c}2.4434 \\
(0.1032)\end{array}$ & $\begin{array}{c}1.6103 \\
(0.0099)\end{array}$ & $\begin{array}{c}0.3835 \\
(0.0846)\end{array}$ & $\begin{array}{c}0.4632 \\
(0.0102)\end{array}$ & $\begin{array}{c}3.1186 \\
(0.1076)\end{array}$ & $\begin{array}{c}1.3623 \\
(0.0048)\end{array}$ & $\begin{array}{c}0.4526 \\
(0.0849)\end{array}$ & $\begin{array}{c}2.5866 \\
(0.1290)\end{array}$ & $\begin{array}{c}1.5811 \\
(0.1082)\end{array}$ & $\begin{array}{c}0.3272 \\
(0.0021)\end{array}$ & $\begin{array}{c}0.4841 \\
(0.0098)\end{array}$ & $\begin{array}{c}3.1236 \\
(0.1023)\end{array}$ & $\begin{array}{l}1.3215 \\
(0.0049)\end{array}$ & $\begin{array}{c}0.4167 \\
(0.0241)\end{array}$ \\
\hline & 100 & $\begin{array}{c}2.5216 \\
(0.0594)\end{array}$ & $\begin{array}{c}1.6195 \\
(0.0037)\end{array}$ & $\begin{array}{c}0.3747 \\
(0.0817)\end{array}$ & $\begin{array}{c}0.5030 \\
(0.0061)\end{array}$ & $\begin{array}{c}3.1034 \\
(0.0709)\end{array}$ & $\begin{array}{c}1.3201 \\
(0.0029)\end{array}$ & $\begin{array}{c}0.4223 \\
(0.0836)\end{array}$ & $\begin{array}{c}2.5129 \\
(0.0973)\end{array}$ & $\begin{array}{c}1.6065 \\
(0.0839)\end{array}$ & $\begin{array}{c}0.3000 \\
(0.0018)\end{array}$ & $\begin{array}{c}0.5027 \\
(0.0060)\end{array}$ & $\begin{array}{c}3.0379 \\
(0.0910)\end{array}$ & $\begin{array}{c}1.3040 \\
(0.0028)\end{array}$ & $\begin{array}{c}0.4191 \\
(0.0163)\end{array}$ \\
\hline \multirow{4}{*}{ QLF } & 20 & $\begin{array}{c}2.4997 \\
(0.1213)\end{array}$ & $\begin{array}{c}1.5489 \\
(0.0202)\end{array}$ & $\begin{array}{c}0.3665 \\
(0.1092)\end{array}$ & $\begin{array}{c}0.4378 \\
(0.0708)\end{array}$ & $\begin{array}{c}2.6539 \\
(0.1224)\end{array}$ & $\begin{array}{c}1.3978 \\
(0.0185)\end{array}$ & $\begin{array}{c}0.3905 \\
(0.1485)\end{array}$ & $\begin{array}{c}2.2865 \\
(0.1128)\end{array}$ & $\begin{array}{c}1.5296 \\
(0.0234)\end{array}$ & $\begin{array}{c}0.3493 \\
(0.0244)\end{array}$ & $\begin{array}{c}0.4431 \\
(0.0647)\end{array}$ & $\begin{array}{c}2.6142 \\
(0.1157)\end{array}$ & $\begin{array}{l}1.4586 \\
(0.0160)\end{array}$ & $\begin{array}{c}0.4503 \\
(0.0071)\end{array}$ \\
\hline & 40 & $\begin{array}{c}2.5161 \\
(0.0609)\end{array}$ & $\begin{array}{l}1.5609 \\
(0.0101)\end{array}$ & $\begin{array}{c}0.3586 \\
(0.0963)\end{array}$ & $\begin{array}{c}0.4687 \\
(0.0312)\end{array}$ & $\begin{array}{c}2.8030 \\
(0.0609)\end{array}$ & $\begin{array}{c}1.3423 \\
(0.0078)\end{array}$ & $\begin{array}{c}0.3912 \\
(0.1229)\end{array}$ & $\begin{array}{c}2.5871 \\
(0.0635)\end{array}$ & $\begin{array}{c}1.5487 \\
(0.0102)\end{array}$ & $\begin{array}{c}0.3221 \\
(0.0167)\end{array}$ & $\begin{array}{c}0.4628 \\
(0.0384)\end{array}$ & $\begin{array}{c}2.8986 \\
(0.0522)\end{array}$ & $\begin{array}{c}1.3841 \\
(0.0060)\end{array}$ & $\begin{array}{c}0.4458 \\
(0.0044)\end{array}$ \\
\hline & 60 & $\begin{array}{c}2.5395 \\
(0.0389)\end{array}$ & $\begin{array}{c}1.5878 \\
(0.0059)\end{array}$ & $\begin{array}{c}0.3518 \\
(0.0423)\end{array}$ & $\begin{array}{c}0.5006 \\
(0.0202)\end{array}$ & $\begin{array}{c}3.0402 \\
(0.0421)\end{array}$ & $\begin{array}{c}1.3264 \\
(0.0047)\end{array}$ & $\begin{array}{c}0.3979 \\
(0.1090)\end{array}$ & $\begin{array}{c}2.4498 \\
(0.0426)\end{array}$ & $\begin{array}{c}1.5673 \\
(0.0069)\end{array}$ & $\begin{array}{c}0.3172 \\
(0.0145)\end{array}$ & $\begin{array}{c}0.4802 \\
(0.0244)\end{array}$ & $\begin{array}{c}2.9842 \\
(0.0377)\end{array}$ & $\begin{array}{c}1.3498 \\
(0.0041)\end{array}$ & $\begin{array}{c}0.4261 \\
(0.0033)\end{array}$ \\
\hline & 100 & $\begin{array}{c}2.5488 \\
(0.0235)\end{array}$ & $\begin{array}{c}1.6028 \\
(0.0036)\end{array}$ & $\begin{array}{c}0.3132 \\
(0.0049)\end{array}$ & $\begin{array}{c}0.5049 \\
(0.0127)\end{array}$ & $\begin{array}{c}3.0721 \\
(0.0247)\end{array}$ & $\begin{array}{c}1.3150 \\
(0.0018)\end{array}$ & $\begin{array}{c}0.4020 \\
(0.0907)\end{array}$ & $\begin{array}{c}2.4861 \\
(0.0243)\end{array}$ & $\begin{array}{c}1.5896 \\
(0.0030)\end{array}$ & $\begin{array}{c}0.3063 \\
(0.0070)\end{array}$ & $\begin{array}{c}0.5084 \\
(0.0119) \\
\end{array}$ & $\begin{array}{c}3.0612 \\
(0.0246)\end{array}$ & $\begin{array}{c}1.3180 \\
(0.0024)\end{array}$ & $\begin{array}{c}0.4071 \\
(0.0021)\end{array}$ \\
\hline
\end{tabular}

$40 \%$ censoring

UP IP

\begin{tabular}{|c|c|c|c|c|c|c|c|c|c|c|c|c|c|c|c|}
\hline $\mathbf{L F}$ & $n$ & $\alpha_{1}=2.5$ & $\beta_{1}=1.6$ & $\lambda_{1}=0.3$ & $p_{1}=0.5$ & $\alpha_{2}=3.0$ & $\beta_{2}=1.3$ & $\lambda_{2}=0.4$ & $\alpha_{1}=2.5$ & $\beta_{1}=1.6$ & $\lambda_{1}=0.3$ & $p_{1}=0.5$ & $\alpha_{2}=3.0$ & $\beta_{2}=1.3$ & $\lambda_{2}=0.4$ \\
\hline \multirow{8}{*}{ SELF } & \multirow{2}{*}{20} & 2.8919 & 1.7084 & 0.3585 & 0.4312 & 3.2158 & 1.3584 & 0.4557 & 2.6422 & 1.5230 & 0.2521 & 0.4366 & 2.8254 & 1.3882 & 0.3519 \\
\hline & & $(0.2990)$ & $(0.1237)$ & $(0.0438)$ & $(0.0162)$ & $(0.2994)$ & $(0.0637)$ & $(0.0591)$ & $(0.2512)$ & $(0.2754)$ & $(0.0091)$ & $(0.0142)$ & $(0.2998)$ & $(0.1736)$ & $(0.0412)$ \\
\hline & \multirow{2}{*}{40} & 2.7424 & 1.6936 & 0.3470 & 0.4617 & 3.0766 & 1.3605 & 0.4424 & 2.5942 & 1.5418 & 0.2976 & 0.4680 & 2.8824 & 1.3467 & 0.3831 \\
\hline & & $(0.2300)$ & $(0.0392)$ & $(0.0402)$ & $(0.0093)$ & $(0.1581)$ & $(0.0337)$ & $(0.0457)$ & $(0.2153)$ & $(0.2425)$ & $(0.0045)$ & $(0.0084)$ & $(0.1759)$ & $(0.1465)$ & $(0.0296)$ \\
\hline & \multirow{2}{*}{60} & 2.5540 & 1.6632 & 0.3407 & 0.4624 & 3.1220 & 1.3777 & 0.4274 & 2.5626 & 1.5961 & 0.2993 & 0.4748 & 2.9157 & 1.3145 & 0.3997 \\
\hline & & $(0.1157)$ & $(0.0248)$ & $(0.0387)$ & $(0.0070)$ & $(0.1046)$ & $(0.0236)$ & $(0.0439)$ & $(0.1267)$ & $(0.1378)$ & $(0.0033)$ & $(0.0061)$ & $(0.1182)$ & $(0.1284)$ & $(0.0233)$ \\
\hline & \multirow{2}{*}{100} & 2.4784 & 1.6082 & 0.3336 & 0.5248 & 3.0180 & 1.4043 & 0.4249 & 2.5245 & 1.6004 & 0.3089 & 0.4874 & 3.0146 & 1.3024 & 0.4003 \\
\hline & & $(0.0987)$ & $(0.0046)$ & $(0.0310)$ & $(0.0035)$ & $(0.0998)$ & $(0.0177)$ & $(0.0388)$ & $(0.0969)$ & $(0.0985)$ & $(0.0024)$ & $(0.0049)$ & $(0.0972)$ & $(0.0870)$ & $(0.0181)$ \\
\hline \multirow{8}{*}{ PLF } & \multirow{2}{*}{20} & 2.9072 & 1.6869 & 0.3987 & 0.4496 & 3.4031 & 1.4914 & & 2.7000 & 1.4959 & 0.2539 & 0.4526 & 2.8574 & 1.3819 & 0.3535 \\
\hline & & $(0.3305)$ & $(0.0634)$ & $(0.1280)$ & $(0.0368)$ & $(0.3745)$ & $(0.1344)$ & $(0.1017)$ & $(0.2461)$ & $(0.2559)$ & $(0.0236)$ & $(0.0320)$ & $(0.2811)$ & $(0.0465)$ & $(0.0418)$ \\
\hline & \multirow{2}{*}{40} & 2.8549 & 1.6304 & 0.3881 & 0.4718 & 3.1817 & 1.4564 & 0.4706 & 2.6861 & 1.5535 & 0.3081 & 0.4770 & 2.9753 & 1.3683 & 0.3898 \\
\hline & & $(0.2251)$ & $(0.0436)$ & $(0.1123)$ & $(0.0299)$ & $(0.2103)$ & $(0.1156)$ & $(0.0964)$ & $(0.1538)$ & $(0.1743)$ & $(0.0112)$ & $(0.0170)$ & $(0.1859)$ & $(0.0232)$ & $(0.0325)$ \\
\hline & \multirow{2}{*}{60} & 2.6322 & 1.6223 & 0.3806 & 0.4699 & 3.1799 & 1.4429 & 0.4546 & 2.5477 & 1.5844 & 0.3068 & 0.5065 & 2.9829 & 1.3417 & 0.3992 \\
\hline & & $(0.1564)$ & $(0.0183)$ & $(0.0998)$ & $(0.0215)$ & $(0.1157)$ & $(0.0130)$ & $(0.0884)$ & $(0.1142)$ & $(0.1186)$ & $(0.0110)$ & $(0.0119)$ & $(0.1062)$ & $(0.0142)$ & $(0.0287)$ \\
\hline & \multirow{2}{*}{100} & 2.4877 & 1.6424 & 0.3724 & 0.4856 & 3.0696 & 1.4003 & 0.4459 & 2.5032 & 1.6088 & 0.3040 & 0.5002 & 3.0453 & 1.3149 & 0.4039 \\
\hline & & $(0.0970)$ & $(0.0106)$ & $(0.0835)$ & $(0.0074)$ & $(0.0865)$ & $(0.0082)$ & $(0.0864)$ & $(0.0856)$ & $(0.0865)$ & $(0.0103)$ & $(0.0098)$ & $(0.0923)$ & $(0.0080)$ & $(0.0179)$ \\
\hline \multirow{8}{*}{ QLF } & \multirow{2}{*}{20} & 2.3005 & 1.5041 & 0.3630 & 0.4143 & 2.8977 & 1.1013 & 0.3922 & 2.2507 & 1.5285 & 0.3165 & 0.4293 & 3.1282 & 1.4260 & 0.4435 \\
\hline & & $(0.1631)$ & $(0.0346)$ & $(0.1098)$ & $(0.1036)$ & $(0.1747)$ & $(0.0346)$ & $(0.1490)$ & $(0.1410)$ & $(0.0377)$ & $(0.0340)$ & $(0.0797)$ & $(0.1591)$ & $(0.0191)$ & $(0.0152)$ \\
\hline & \multirow{2}{*}{40} & 2.3472 & 1.5464 & 0.3536 & 0.4640 & 2.9048 & 1.2741 & 0.3935 & 2.3767 & 1.5409 & 0.3120 & 0.4611 & 3.0614 & 1.3857 & 0.4354 \\
\hline & & $(0.0816)$ & $(0.0264)$ & $(0.1048)$ & $(0.0420)$ & $(0.0820)$ & $(0.0187)$ & $(0.1449)$ & $(0.0742)$ & $(0.0171)$ & $(0.0235)$ & $(0.0418)$ & $(0.0907)$ & $(0.0121)$ & $(0.0143)$ \\
\hline & \multirow{2}{*}{60} & 2.4336 & 1.5816 & 0.3498 & 0.4974 & 3.0568 & 1.2936 & 0.3981 & 2.4887 & 1.5690 & 0.3010 & 0.5011 & 3.0440 & 1.3450 & 0.4281 \\
\hline & & $(0.0554)$ & $(0.0114)$ & $(0.0633)$ & $(0.0255)$ & $(0.0601)$ & $(0.0173)$ & $(0.1118)$ & $(0.0458)$ & $(0.0092)$ & $(0.0110)$ & $(0.0259)$ & $(0.0679)$ & $(0.0096)$ & $(0.0075)$ \\
\hline & \multirow{2}{*}{100} & 2.5018 & 1.6086 & 0.3134 & 0.5043 & 3.0078 & 1.3017 & 0.4001 & 2.5355 & 1.5910 & 0.3000 & 0.5197 & 3.0152 & 1.3257 & 0.4065 \\
\hline & & $(0.0380)$ & $(0.0037)$ & $(0.0066)$ & $(0.0131)$ & $(0.0328)$ & $(0.0078)$ & $(0.0943)$ & $(0.0327)$ & $(0.0051)$ & $(0.0097)$ & $(0.0196)$ & $(0.0512)$ & $(0.0058)$ & $(0.0040)$ \\
\hline
\end{tabular}


Table A.2. Bayes Estimates (BEs) of mixture for two components of Transmuted Weibull Distribution (TWD) along with Posterior Risks (PRs) (in parentheses) under Uniform Prior (UP) and Informative Prior (IP).

\begin{tabular}{|c|c|c|c|c|c|c|c|c|c|c|c|c|c|c|c|}
\hline \multirow[b]{3}{*}{ LF } & \multicolumn{15}{|c|}{$20 \%$ censoring } \\
\hline & \multicolumn{8}{|c|}{ UP } & \multicolumn{7}{|c|}{ IP } \\
\hline & $n$ & $\alpha_{1}=2$ & $\beta_{1}=1.4$ & $\lambda_{1}=0.4$ & $p_{1}=0.7$ & $\alpha_{2}=1.5$ & $\beta_{2}=1.3$ & $\lambda_{2}=0.6$ & $\alpha_{1}=2$ & $\beta_{1}=1.4$ & $\lambda_{1}=0.4$ & $p_{1}=0.7$ & $\alpha_{2}=1.5$ & $\beta_{2}=1.3$ & $\lambda_{2}=0.6$ \\
\hline \multirow{7}{*}{ SELF } & 20 & 1.7365 & 1.3246 & 0.4419 & 0.6094 & 1.4573 & 1.2451 & 0.6276 & 1.6826 & 1.5461 & 0.3212 & 0.6412 & 1.2515 & 1.2084 & 0.5034 \\
\hline & 20 & $(0.2696)$ & $(0.0466)$ & $(0.0177)$ & $(0.0126)$ & $(0.3051)$ & $(0.1226)$ & $(0.0355)$ & $(0.2285)$ & $(0.1369)$ & $(0.0431)$ & $(0.0110)$ & $(0.2241)$ & $(0.1559)$ & $(0.0338)$ \\
\hline & 40 & 1.8127 & 1.3542 & 0.4320 & 0.6166 & 1.4633 & 1.2452 & 0.6090 & 1.7881 & 1.4882 & 0.3667 & 0.6617 & 1.3838 & 1.2464 & 0.5516 \\
\hline & & $(0.1534)$ & $(0.0110)$ & $(0.0086)$ & $(0.0068)$ & $(0.1583)$ & $(0.1210)$ & $(0.0317)$ & $(0.1334)$ & $(0.0965)$ & $(0.0168)$ & $(0.0061)$ & $(0.1566)$ & $(0.1230)$ & $(0.0214)$ \\
\hline & 60 & 1.9367 & 1.3875 & 0.4200 & 0.6595 & 1.4840 & 1.2769 & 0.6063 & 1.9234 & 1.4599 & 0.3694 & 0.6901 & 1.4654 & 1.2652 & 0.5681 \\
\hline & & $(0.1103)$ & $(0.0049)$ & $(0.0072)$ & $(0.0044)$ & $(0.1184)$ & $(0.0536)$ & $(0.0303)$ & $(0.1011)$ & $(0.0472)$ & $(0.0161)$ & $(0.0040)$ & $(0.1144)$ & $(0.1036)$ & $(0.0208)$ \\
\hline & 100 & $\begin{array}{c}2.0420 \\
(0.0729)\end{array}$ & $\begin{array}{c}1.4087 \\
(0.0023)\end{array}$ & $\begin{array}{c}0.4143 \\
(0.0056)\end{array}$ & $\begin{array}{c}0.6812 \\
(0.0026)\end{array}$ & $\begin{array}{c}1.5068 \\
(0.0838)\end{array}$ & $\begin{array}{c}1.2948 \\
(0.0320)\end{array}$ & $\begin{array}{c}0.5925 \\
(0.0276)\end{array}$ & $\begin{array}{c}2.0459 \\
(0.0715)\end{array}$ & $\begin{array}{c}1.4277 \\
(0.0216)\end{array}$ & $\begin{array}{c}0.4074 \\
(0.0135)\end{array}$ & $\begin{array}{c}0.7125 \\
(0.0025)\end{array}$ & $\begin{array}{c}1.4918 \\
(0.0829)\end{array}$ & $\begin{array}{c}1.2982 \\
(0.0929)\end{array}$ & $\begin{array}{c}0.5998 \\
(0.0132)\end{array}$ \\
\hline \multirow{8}{*}{ PLF } & 20 & 1.8125 & 1.4775 & 0.4615 & 0.6197 & 1.5585 & 1.2390 & 0.6553 & 1.7492 & 1.5796 & 0.3410 & 0.6497 & 1.3380 & 1.4364 & 0.5170 \\
\hline & & $(0.1519)$ & $(0.0612)$ & $(0.0391)$ & $(0.0205)$ & $(0.2024)$ & $(0.1590)$ & $(0.0554)$ & $(0.1332)$ & $(0.1436)$ & $(0.0696)$ & $(0.0170)$ & ) $(0.1731)$ & $(0.1266)$ & $(0.0571)$ \\
\hline & 40 & 1.8545 & 1.4615 & 0.4532 & 0.6221 & 1.5479 & 1.2556 & 0.6341 & 1.8250 & 1.4951 & 0.3890 & 0.6663 & 1.4393 & 1.3556 & 0.5706 \\
\hline & 40 & $(0.0836)$ & $(0.0146)$ & $(0.0223)$ & $(0.0109)$ & $(0.1091)$ & $(0.1206)$ & $(0.0502)$ & $(0.0738)$ & $(0.0825)$ & $(0.0446)$ & $(0.0092)$ & $(0.1110)$ & $(0.1085)$ & $(0.0381)$ \\
\hline & 60 & 1.9650 & 1.4309 & 0.4399 & 0.6628 & 1.5255 & 1.2881 & 0.6308 & 1.9496 & 1.4530 & 0.3905 & 0.6930 & 1.4665 & 1.3394 & 0.5876 \\
\hline & & $(0.0565)$ & $(0.0067)$ & $(0.0199)$ & $(0.0067)$ & $(0.0831)$ & $(0.0838)$ & $(0.0489)$ & $(0.0522)$ & $(0.0562)$ & $(0.0423)$ & $(0.0058)$ & $(0.0825)$ & $(0.0983)$ & $(0.0374)$ \\
\hline & 100 & 2.0598 & 1.4130 & 0.4142 & 0.6831 & 1.4951 & 1.3014 & 0.6153 & 2.0633 & 1.4291 & 0.4129 & 0.7042 & 1.4905 & 1.3116 & 0.6108 \\
\hline & 100 & $(0.0356)$ & $(0.0031)$ & $(0.0086)$ & $(0.0038)$ & $(0.0566)$ & $(0.0632)$ & $(0.0457)$ & $(0.0348)$ & $(0.0288)$ & $(0.0310)$ & $(0.0034)$ & $(0.0673)$ & $(0.0773)$ & $(0.0218)$ \\
\hline \multirow{8}{*}{ QLF } & 20 & 1.7749 & 1.3447 & 0.4947 & 0.6253 & 1.2353 & 1.4082 & 0.5660 & 1.7364 & 1.5418 & 0.3776 & 0.6582 & 1.4199 & 1.2108 & 0.5256 \\
\hline & & $(0.0917)$ & $(0.0287)$ & $(0.0882)$ & $(0.0335)$ & $(0.1956)$ & $(0.1210)$ & $(0.1108)$ & $(0.0795)$ & $(0.0229)$ & $(0.0871)$ & $(0.0272)$ & ) $(0.1969)$ & $(0.2121)$ & $(0.0557)$ \\
\hline & 40 & 1.9107 & 1.3768 & 0.4799 & 0.6436 & 1.3125 & 1.3579 & 0.6073 & 1.9821 & 1.4887 & 0.3914 & 0.6713 & 1.4426 & 1.2547 & 0.5422 \\
\hline & & $(0.0513)$ & $(0.0131)$ & $(0.0786)$ & $(0.0198)$ & $(0.0831)$ & $(0.0542)$ & $(0.0615)$ & $(0.0416)$ & $(0.0098)$ & $(0.0633)$ & $(0.0141)$ & ) $(0.1000)$ & $(0.1471)$ & $(0.0445)$ \\
\hline & 60 & 2.0327 & 1.3803 & 0.4476 & 0.6661 & 1.4826 & 1.3312 & 0.6014 & 1.9914 & 1.4490 & 0.3944 & 0.6857 & 1.4751 & 1.2822 & 0.5576 \\
\hline & & $(0.0303)$ & $(0.0068)$ & $(0.0206)$ & $(0.0105)$ & $(0.0653)$ & $(0.0433)$ & $(0.0592)$ & $(0.0299)$ & $(0.0068)$ & $(0.0547)$ & $(0.0115)$ & $(0.0605)$ & $(0.1097)$ & $(0.0387)$ \\
\hline & 100 & 2.0252 & 1.4071 & 0.4244 & 0.6917 & 1.5158 & 1.3113 & 0.6001 & 2.0301 & 1.4150 & 0.4068 & 0.6919 & 1.4950 & 1.3062 & 0.6086 \\
\hline & & $(0.0180)$ & 040) & $(0.0197)$ & $(0.0064)$ & $(0.0387)$ & $(0.0210)$ & $(0.0545)$ & $(0.0171)$ & $(0.0036)$ & $(0.0487)$ & $(0.0073)$ & ) $(0.0406)$ & $(0.0876)$ & $(0.0181)$ \\
\hline
\end{tabular}

$40 \%$ censoring

UP

IP

\begin{tabular}{|c|c|c|c|c|c|c|c|c|c|c|c|c|c|c|c|}
\hline $\mathbf{L F}$ & $n$ & $\alpha_{1}=2$ & $\beta_{1}=1.4$ & $\lambda_{1}=0.4$ & $p_{1}=0.7$ & $\alpha_{2}=1.5$ & $\beta_{2}=1.3$ & $\lambda_{2}=0.6$ & $\alpha_{1}=2$ & $\beta_{1}=1.4$ & $\lambda_{1}=0.4$ & $p_{1}=0.7$ & $\alpha_{2}=1$ & $\beta_{2}=1.3$ & $\lambda_{2}=0.6$ \\
\hline \multirow{8}{*}{ SELF } & \multirow{2}{*}{20} & 1.8528 & 70 & 610 & 0.661 & 1360 & 4961 & 0.5788 & 1.5916 & 1. & 0.3 & 0. & $1.2 \mathrm{~s}$ & 1.4670 & 7 \\
\hline & & $(0.2$ & $(0.0533)$ & $(0.0278)$ & $(0.0174)$ & $(0.3355)$ & $(0.1396)$ & $(0.0449)$ & $(0.2658)$ & $(0.1511)$ & $(0.0514)$ & $(0.0144)$ & $(0.2794)$ & $(0.1598)$ & $(0.0377)$ \\
\hline & \multirow{2}{*}{40} & 1.9251 & 1.4812 & 0.4422 & 0.651 & 1.4654 & 1.405 & 0.581 & 1.7675 & 1.4867 & 03614 & 0.6357 & 1.37 & 1.4296 & 0.5682 \\
\hline & & $(0.1606)$ & $(0.0453)$ & $(0.0263)$ & $(0.0081)$ & $(0.1659)$ & $(0.1245)$ & $(0.0431)$ & $(0.1769)$ & $(0.1098)$ & $(0.0234)$ & $(0.0085)$ & $(0.1673)$ & $(0.1286)$ & $(0.0235)$ \\
\hline & \multirow{2}{*}{6} & 2.0264 & 1.4491 & 0.4305 & 0.6840 & 1.4987 & 1.3519 & 0.5605 & 1.9690 & 1.4462 & 0.3809 & 0.6684 & 1.4404 & 1.3572 & 0.5803 \\
\hline & & $(0.1249)$ & $(0.0150)$ & $(0.0247)$ & $(0.0057)$ & $(0.1202)$ & $(0.0692)$ & $(0.0415)$ & $(0.1180)$ & $(0.0694)$ & $(0.0194)$ & $(0.0054)$ & $(0.1289)$ & $(0.1057)$ & $(0.0214)$ \\
\hline & \multirow{2}{*}{100} & 2.0 & 1.4 & 0.42 & 0. & 1. & 1.32 & 0. & 1.99 & 1.4 & 0.40 & 0.6 & 1.5 & 1.3254 & 0.6028 \\
\hline & & $(0.0959)$ & $(0.0087)$ & $(0.0153)$ & $(0.0031)$ & $(0.0957)$ & $(0.0345)$ & $(0.0394)$ & $(0.0848)$ & $(0.0475)$ & $(0.0149)$ & $(0.0035)$ & $(0.0973)$ & $(0.0963)$ & $(0.0154)$ \\
\hline
\end{tabular}

$\begin{array}{lllllllllllllll}20 & 1.8451 & 1.6202 & 0.4902 & 0.6743 & 1.6117 & 1.3651 & 0.6164 & 1.6730 & 1.4663 & 0.3529 & 0.6254 & 1.3978 & 1.4782 & 0.5567\end{array}$

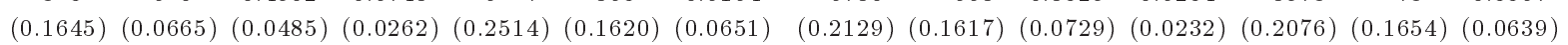

$\begin{array}{lllllllllllllll}40 & 1.9738 & 1.5674 & 0.4711 & 0.6577 & 1.5405 & 1.3457 & 0.6078 & 1.8168 & 1.4493 & 0.3643 & 0.6476 & 1.4308 & 1.3831 & 0.5794\end{array}$

(0.0974) (0.0323) (0.0378) (0.0124) (0.1503) (0.1306) (0.0533) (0.1187) (0.1257) (0.0584) (0.0140) (0.1194) $(0.1222)(0.0420)$

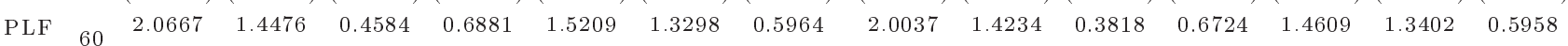
60 (0.0806) (0.0170) (0.0257) (0.0083) (0.1243) $(0.1210)(0.0517)(0.0895)(0.0939)(0.0441)(0.0080)(0.1010)(0.1097)(0.0393)$ $100 \quad \begin{array}{llllllllllllll}2.0943 & 1.4165 & 0.4253 & 0.6936 & 1.5045 & 1.3098 & 0.5920 & 2.0209 & 1.4080 & 0.4000 & 0.6931 & 1.5019 & 1.3862 & 0.6056\end{array}$ $100 \begin{array}{llllllllllllllll}(0.0460) & (0.0102) & (0.0174) & (0.0045) & (0.0979) & (0.0989) & (0.0486) & (0.0572) & (0.0685) & (0.0326) & (0.0061) & (0.0867) & (0.0916) & (0.0256)\end{array}$

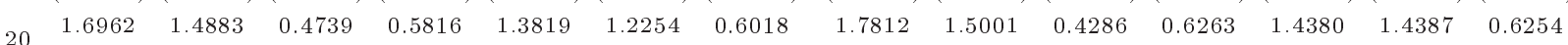

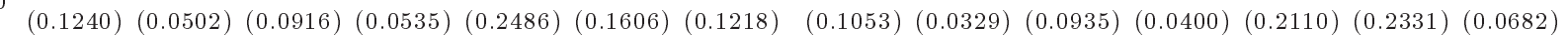

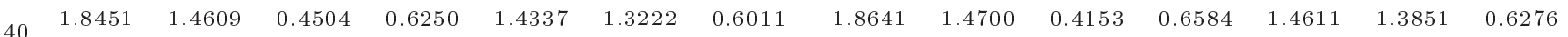

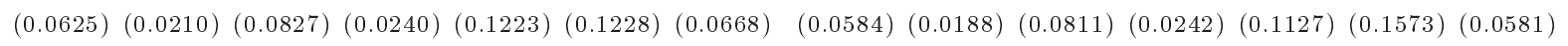

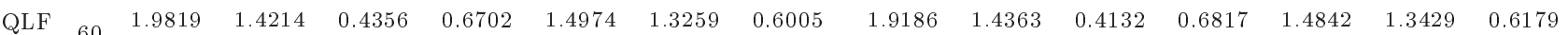
$60(0.0407)(0.0114)(0.0211)(0.0140)(0.0791)(0.0787)(0.0638)(0.0362)(0.0109)(0.0780)(0.0145)(0.0968)(0.1206)(0.0468)$ $\begin{array}{llllllllllllll}2.0711 & 1.4037 & 0.4017 & 0.7028 & 1.5075 & 1.3029 & 0.5973 & 1.9764 & 1.4054 & 0.4000 & 0.7005 & 1.5202 & 1.3153 & 0.6073\end{array}$

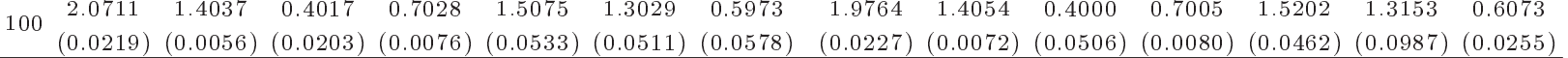


Table A.3. 95\% Bayesian credible intervals of mixture for two components of Transmuted Weibull Distribution (TWD) using Uniform Prior (UP) and Informative Prior (IP) with hyperparameters are $a_{1}=1, a_{2}=0.5, b_{1}=2, b_{2}=1, c_{1}=0.5$, $c_{2}=1.5, d_{1}=1, d_{2}=2, e_{1}=1, e_{2}=1, l_{1}=0.1$, and $l_{2}=0.2$.

\begin{tabular}{|c|c|c|c|c|c|c|}
\hline \multirow[t]{2}{*}{ Censoring rate } & \multirow[t]{2}{*}{ Size } & \multirow[t]{2}{*}{ Parameters } & \multicolumn{2}{|c|}{ UP } & \multicolumn{2}{|c|}{ IP } \\
\hline & & & Lower limit & Upper limit & Lower limit & Upper limit \\
\hline \multirow{7}{*}{$20 \%$} & \multirow{14}{*}{20} & $\alpha_{1}=2.5$ & 1.4024 & 13.8814 & 1.9556 & 8.8013 \\
\hline & & $\alpha_{2}=3.0$ & 1.5739 & 7.6740 & 2.1231 & 14.1630 \\
\hline & & $\beta_{1}=1.6$ & 0.7097 & 2.1776 & 0.8392 & 2.2626 \\
\hline & & $\beta_{2}=1.3$ & 0.7231 & 2.1907 & 0.7660 & 2.2391 \\
\hline & & $\lambda_{1}=0.3$ & 0.2426 & 0.8693 & 0.3102 & 0.4916 \\
\hline & & $\lambda_{2}=0.4$ & 0.3099 & 0.9310 & 0.4552 & 0.5467 \\
\hline & & $p_{1}=0.5$ & 0.2663 & 2.1117 & 0.3179 & 1.4747 \\
\hline \multirow{7}{*}{$40 \%$} & & $\alpha_{1}=2.5$ & 1.4336 & 14.7699 & 1.9684 & 9.9844 \\
\hline & & $\alpha_{2}=3.0$ & 1.6647 & 8.3043 & 2.3110 & 15.4317 \\
\hline & & $\beta_{1}=1.6$ & 0.7177 & 4.4872 & 0.8487 & 5.7680 \\
\hline & & $\beta_{2}=1.3$ & 0.7352 & 4.3795 & 0.7769 & 4.7679 \\
\hline & & $\lambda_{1}=0.3$ & 0.2521 & 0.8759 & 0.3438 & 0.9665 \\
\hline & & $\lambda_{2}=0.4$ & 0.3389 & 0.9450 & 0.4561 & 1.0698 \\
\hline & & $p_{1}=0.5$ & 0.2736 & 2.2102 & 0.3224 & 1.7848 \\
\hline \multirow{8}{*}{$20 \%$} & \multirow{14}{*}{40} & $\alpha_{1}=2.5$ & 1.4012 & 12.3951 & 1.1265 & 8.6167 \\
\hline & & $\alpha_{2}=3.0$ & 1.0164 & 6.9280 & 1.3375 & 9.9317 \\
\hline & & $\beta_{1}=1.6$ & 0.5029 & 2.1642 & 0.5760 & 2.2518 \\
\hline & & $\beta_{2}=1.3$ & 0.4676 & 2.1694 & 0.5158 & 2.2191 \\
\hline & & $\lambda_{1}=0.3$ & 0.2348 & 0.8653 & 0.3011 & 0.3737 \\
\hline & & $\lambda_{2}=0.4$ & 0.3076 & 0.9205 & 0.4086 & 0.5000 \\
\hline & & $p_{1}=0.5$ & 0.2511 & 1.8660 & 0.2102 & 1.3802 \\
\hline & & $\alpha_{1}=2.5$ & 1.4073 & 14.5029 & 1.3358 & 8.9419 \\
\hline \multirow{6}{*}{$40 \%$} & & $\alpha_{2}=3.0$ & 1.0631 & 8.2743 & 1.5659 & 13.5041 \\
\hline & & $\beta_{1}=1.6$ & 0.5146 & 4.1274 & 0.6094 & 5.2276 \\
\hline & & $\beta_{2}=1.3$ & 0.4944 & 4.8022 & 0.5269 & 4.5021 \\
\hline & & $\lambda_{1}=0.3$ & 0.2351 & 0.8660 & 0.3057 & 0.4977 \\
\hline & & $\lambda_{2}=0.4$ & 0.3332 & 0.9366 & 0.4098 & 0.5079 \\
\hline & & $p_{1}=0.5$ & 0.2728 & 2.1452 & 0.2257 & 1.7002 \\
\hline \multirow{6}{*}{$20 \%$} & \multirow{14}{*}{60} & $\alpha_{1}=2.5$ & 0.9431 & 12.1943 & 0.6911 & 8.5076 \\
\hline & & $\alpha_{2}=3.0$ & 1.0106 & 6.2809 & 1.2292 & 9.5548 \\
\hline & & $\beta_{1}=1.6$ & 0.3780 & 1.7228 & 0.4210 & 2.2097 \\
\hline & & $\beta_{2}=1.3$ & 0.3568 & 1.5870 & 0.3834 & 2.1807 \\
\hline & & $\lambda_{1}=0.3$ & 0.2240 & 0.8355 & 0.3002 & 0.3284 \\
\hline & & $\lambda_{2}=0.4$ & 0.3099 & 0.9124 & 0.4060 & 0.4536 \\
\hline \multirow{8}{*}{$40 \%$} & & $p_{1}=0.5$ & 0.1599 & 1.8515 & 0.1609 & 1.3089 \\
\hline & & $\alpha_{1}=2.5$ & 0.9592 & 14.5153 & 0.7058 & 8.9087 \\
\hline & & $\alpha_{2}=3.0$ & 1.0530 & 8.7726 & 1.2757 & 9.9611 \\
\hline & & $\beta_{1}=1.6$ & 0.3823 & 5.1488 & 0.4336 & 5.1898 \\
\hline & & $\beta_{2}=1.3$ & 0.3708 & 5.5830 & 0.3935 & 4.3529 \\
\hline & & $\lambda_{1}=0.3$ & 0.2264 & 0.8381 & 0.3025 & 0.3951 \\
\hline & & $\lambda_{2}=0.4$ & 0.3270 & 0.8659 & 0.4095 & 0.4957 \\
\hline & & $p_{1}=0.5$ & 0.1808 & 2.1218 & 0.1781 & 1.6148 \\
\hline \multirow{6}{*}{$20 \%$} & \multirow{14}{*}{100} & $\alpha_{1}=2.5$ & 0.4291 & 11.2170 & 0.3840 & 8.5019 \\
\hline & & $\alpha_{2}=3.0$ & 0.5522 & 5.8514 & 0.4947 & 9.0473 \\
\hline & & $\beta_{1}=1.6$ & 0.2249 & 1.6703 & 0.2565 & 2.2021 \\
\hline & & $\beta_{2}=1.3$ & 0.2105 & 1.5804 & 0.2498 & 2.0233 \\
\hline & & $\lambda_{1}=0.3$ & 0.2219 & 0.8312 & 0.2967 & 0.3240 \\
\hline & & $\lambda_{2}=0.4$ & 0.3064 & 0.9093 & 0.4042 & 0.4503 \\
\hline \multirow{8}{*}{$40 \%$} & & $p_{1}=0.5$ & 0.0960 & 1.3365 & 0.1193 & 1.0372 \\
\hline & & $\alpha_{1}=2.5$ & 0.4342 & 13.8626 & 0.3992 & 8.7897 \\
\hline & & $\alpha_{2}=3.0$ & 0.5631 & 7.9133 & 0.6913 & 9.5036 \\
\hline & & $\beta_{1}=1.6$ & 0.2436 & 5.0806 & 0.4279 & 4.8949 \\
\hline & & $\beta_{2}=1.3$ & 0.2396 & 5.0557 & 0.3829 & 3.0396 \\
\hline & & $\lambda_{1}=0.3$ & 0.2246 & 0.8325 & 0.3008 & 0.3945 \\
\hline & & $\lambda_{2}=0.4$ & 0.3104 & 0.8101 & 0.4088 & 0.4862 \\
\hline & & $p_{1}=0.5$ & 0.0981 & 2.0300 & 0.1600 & 1.2988 \\
\hline
\end{tabular}


Table A.4. 95\% Bayesian credible intervals of mixture for two components of Transmuted Weibull Distribution (TWD) using Uniform Prior (UP) and Informative Prior (IP) with hyperparameters are $a_{1}=0.5, a_{2}=1, b_{1}=1, b_{2}=2, c_{1}=1.5$, $c_{2}=0.5, d_{1}=2, d_{2}=1, e_{1}=0.5, e_{2}=1, l_{1}=0.1$ and $l_{2}=0.2$.

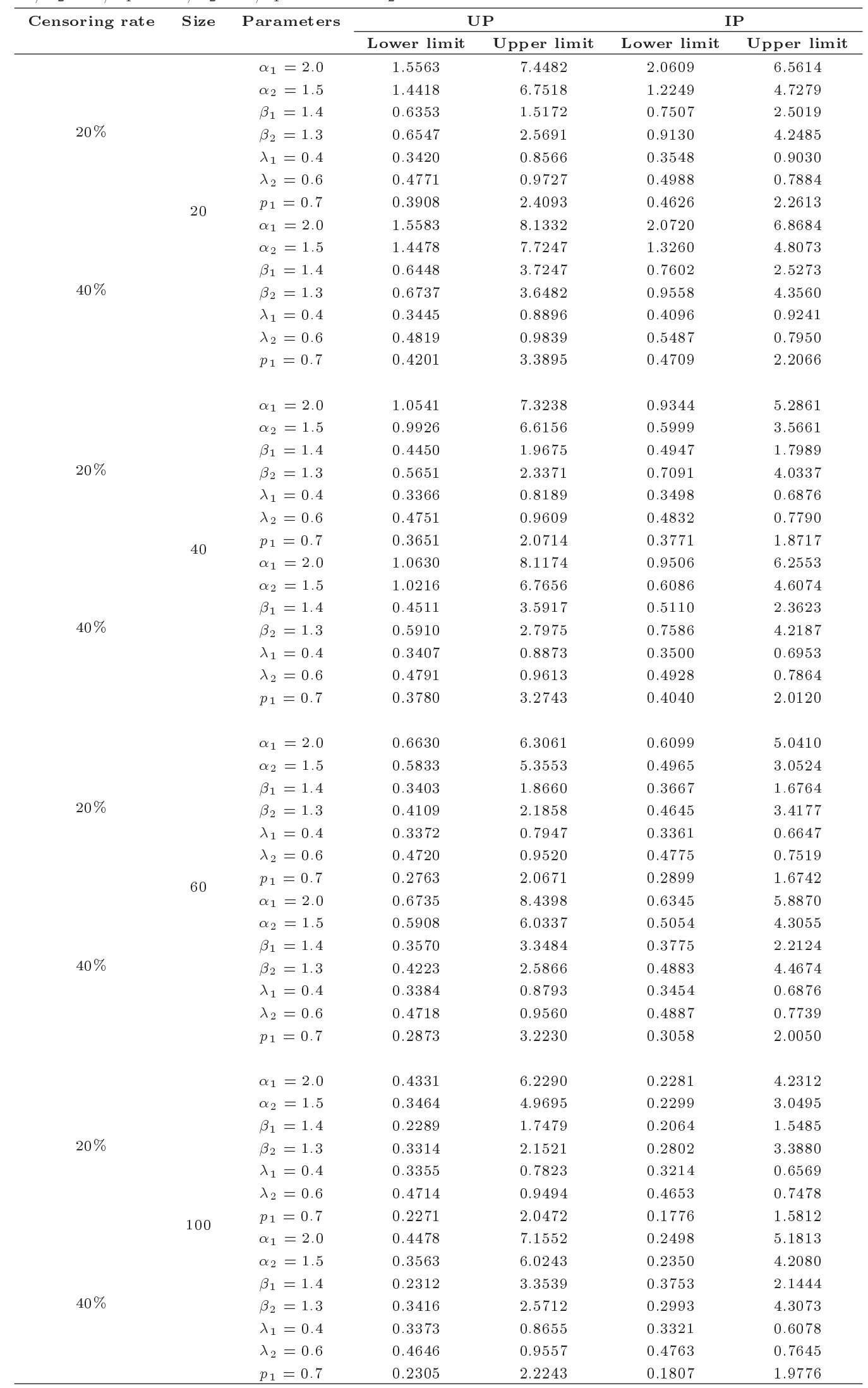


Table A.5. Measurements of hole and sheet thickness (12 $\mathrm{mm}$ and $3.15 \mathrm{~mm})$.

\begin{tabular}{ll}
\hline \multicolumn{1}{c}{ Subpopulation-I } & \multicolumn{1}{c}{ Subpopulation-II } \\
\hline $0.04,0.06,0.12,0.22,0.08,0.26,0.14,0,0.08,0.32$ & $0.02,0.14,0.08,0.12,0.24,0.04,0.16,0.26,0.28$, \\
$0.14,0.16,0.12,0.24,0.16,0.08,0.16,0.32,0.24,0.22$ & $0.24,0.22,0.18,0.32,0.14,0.24,0.16,0.18,0.16$, \\
$0.24,0.02,0.22,0.06,0.14,0.26$ & $0.12,0.06,0.18,0.14,0.04,0.18,0.16$ \\
\hline
\end{tabular}

Table A.6. Bayes Estimates (BEs) of mixture for two components of Transmuted Weibull Distribution (TWD) along with Posterior Risks (PRs) (in parentheses) under Uniform Prior (UP) and Informative Prior (IP).

\begin{tabular}{|c|c|c|c|c|c|c|c|c|c|c|c|c|c|c|}
\hline \multirow[b]{3}{*}{ LF } & \multicolumn{13}{|c|}{$20 \%$ censoring } & \\
\hline & \multicolumn{7}{|c|}{ UP } & \multicolumn{7}{|c|}{ IP } \\
\hline & $\alpha_{1}=2.5$ & $\beta_{1}=1.6$ & $\lambda_{1}=0.3$ & $p_{1}=0.5$ & $\alpha_{2}=3.0$ & $\beta_{2}=1.3$ & $\lambda_{2}=0.4$ & $\alpha_{1}=2.5$ & $\beta_{1}=1.6$ & $\lambda_{1}=0.3$ & $p_{1}=0.5$ & $\alpha_{2}=3.0$ & $\beta_{2}=1.3$ & $\lambda_{2}=0.4$ \\
\hline SELF & $\begin{array}{c}2.4811 \\
(0.0121)\end{array}$ & $\begin{array}{c}1.5778 \\
(0.0213)\end{array}$ & $\begin{array}{c}0.2998 \\
(0.0132)\end{array}$ & $\begin{array}{c}0.4993 \\
(0.0062)\end{array}$ & $\begin{array}{c}3.0539 \\
(0.0129)\end{array}$ & $\begin{array}{c}1.2854 \\
(0.0222)\end{array}$ & $\begin{array}{c}0.3998 \\
(0.0134)\end{array}$ & $\begin{array}{c}2.4947 \\
(0.0109)\end{array}$ & $\begin{array}{c}1.5813 \\
(0.0179)\end{array}$ & $\begin{array}{c}0.2977 \\
(0.0002)\end{array}$ & $\begin{array}{c}0.4970 \\
(0.0062)\end{array}$ & $\begin{array}{c}3.0810 \\
(0.0112)\end{array}$ & $\begin{array}{c}1.2925 \\
(0.0181)\end{array}$ & $\begin{array}{c}0.4177 \\
(0.0075)\end{array}$ \\
\hline PLF & $\begin{array}{c}2.4935 \\
(0.0248)\end{array}$ & $\begin{array}{c}1.6267 \\
(0.0258)\end{array}$ & $\begin{array}{c}0.3207 \\
(0.0428)\end{array}$ & $\begin{array}{c}0.4956 \\
(0.0126)\end{array}$ & $\begin{array}{c}3.0180 \\
(0.0252)\end{array}$ & $\begin{array}{c}1.2995 \\
(0.0280)\end{array}$ & $\begin{array}{c}0.4214 \\
(0.0431)\end{array}$ & $\begin{array}{c}2.4861 \\
(0.0227)\end{array}$ & $\begin{array}{c}1.5819 \\
(0.0211)\end{array}$ & $\begin{array}{c}0.2981 \\
(0.0007)\end{array}$ & $\begin{array}{c}0.4933 \\
(0.0126)\end{array}$ & $\begin{array}{c}2.9925 \\
(0.0230)\end{array}$ & $\begin{array}{c}1.2730 \\
(0.0211)\end{array}$ & $\begin{array}{c}0.4160 \\
(0.0166)\end{array}$ \\
\hline QLF & $\begin{array}{c}2.4315 \\
(0.0540)\end{array}$ & $\begin{array}{c}1.6129 \\
(0.0468)\end{array}$ & $\begin{array}{c}0.3016 \\
(0.0883)\end{array}$ & $\begin{array}{c}0.4823 \\
(0.0289)\end{array}$ & $\begin{array}{c}3.0538 \\
(0.0540)\end{array}$ & $\begin{array}{c}1.3129 \\
(0.0526)\end{array}$ & $\begin{array}{c}0.4013 \\
(0.0894)\end{array}$ & $\begin{array}{c}2.4890 \\
(0.0519)\end{array}$ & $\begin{array}{c}1.5917 \\
(0.0320)\end{array}$ & $\begin{array}{c}0.2951 \\
(0.0052)\end{array}$ & $\begin{array}{c}0.4895 \\
(0.0297)\end{array}$ & $\begin{array}{c}2.9848 \\
(0.0501)\end{array}$ & $\begin{array}{c}1.2802 \\
(0.0324)\end{array}$ & $\begin{array}{c}0.4139 \\
(0.0388)\end{array}$ \\
\hline \multicolumn{15}{|c|}{$40 \%$ censoring } \\
\hline & \multicolumn{7}{|c|}{ UP } & \multicolumn{7}{|c|}{ IP } \\
\hline $\mathbf{L F}$ & $\alpha_{1}=2.5$ & $\beta_{1}=1.6$ & $\lambda_{1}=0.3$ & $p_{1}=0.5$ & $\alpha_{2}=3.0$ & $\beta_{2}=1.3$ & $\lambda_{2}=0.4$ & $\alpha_{1}=2.5$ & $\beta_{1}=1.6$ & $\lambda_{1}=0.3$ & $p_{1}=0.5$ & $\alpha_{2}=3.0$ & $\beta_{2}=1.3$ & $\lambda_{2}=0.4$ \\
\hline SELF & $\begin{array}{c}2.4636 \\
(0.0137)\end{array}$ & $\begin{array}{c}1.5911 \\
(0.0287)\end{array}$ & $\begin{array}{c}0.3013 \\
(0.0137)\end{array}$ & $\begin{array}{c}0.5129 \\
(0.0078)\end{array}$ & $\begin{array}{c}3.0274 \\
(0.0146)\end{array}$ & $\begin{array}{c}1.3116 \\
(0.0287)\end{array}$ & $\begin{array}{c}0.3989 \\
(0.0138)\end{array}$ & $\begin{array}{c}2.4911 \\
(0.0114)\end{array}$ & $\begin{array}{c}1.5914 \\
(0.0237)\end{array}$ & $\begin{array}{c}0.3196 \\
(0.0074)\end{array}$ & $\begin{array}{c}0.5148 \\
(0.0076)\end{array}$ & $\begin{array}{c}2.9915 \\
(0.0127)\end{array}$ & $\begin{array}{c}1.2990 \\
(0.0275)\end{array}$ & $\begin{array}{c}0.4086 \\
(0.0094)\end{array}$ \\
\hline PLF & $\begin{array}{c}2.4781 \\
(0.0290)\end{array}$ & $\begin{array}{c}1.6272 \\
(0.0312)\end{array}$ & $\begin{array}{c}0.3222 \\
(0.0437)\end{array}$ & $\begin{array}{c}0.5205 \\
(0.0152)\end{array}$ & $\begin{array}{c}3.0408 \\
(0.0268)\end{array}$ & $\begin{array}{c}1.2855 \\
(0.0292)\end{array}$ & $\begin{array}{c}0.4205 \\
(0.0436)\end{array}$ & $\begin{array}{c}2.4636 \\
(0.0249)\end{array}$ & $\begin{array}{c}1.5974 \\
(0.0245)\end{array}$ & $\begin{array}{c}0.3201 \\
(0.0209)\end{array}$ & $\begin{array}{c}0.5221 \\
(0.0147)\end{array}$ & $\begin{array}{c}2.9654 \\
(0.0277)\end{array}$ & $\begin{array}{c}1.2821 \\
(0.0266)\end{array}$ & $\begin{array}{c}0.4567 \\
(0.0173)\end{array}$ \\
\hline QLF & $\begin{array}{c}2.4546 \\
(0.0676)\end{array}$ & $\begin{array}{c}1.6337 \\
(0.0477)\end{array}$ & $\begin{array}{c}0.3043 \\
(0.0896)\end{array}$ & $\begin{array}{c}0.4884 \\
(0.0362)\end{array}$ & $\begin{array}{c}3.0721 \\
(0.0693)\end{array}$ & $\begin{array}{c}1.2721 \\
(0.0551)\end{array}$ & $\begin{array}{c}0.3996 \\
(0.0919)\end{array}$ & $\begin{array}{c}2.4002 \\
(0.0601)\end{array}$ & $\begin{array}{c}1.5905 \\
(0.0337)\end{array}$ & $\begin{array}{c}0.3057 \\
(0.0061)\end{array}$ & $\begin{array}{c}0.4823 \\
(0.0336)\end{array}$ & $\begin{array}{c}2.9577 \\
(0.0656)\end{array}$ & $\begin{array}{c}1.2955 \\
(0.0341)\end{array}$ & $\begin{array}{c}0.4149 \\
(0.0397)\end{array}$ \\
\hline
\end{tabular}

Table A.7. 95\% Bayesian credible intervals of mixture of the two components of Transmuted Weibull Distribution (TWD) using Uniform Prior (UP) and Informative Prior (IP).

\begin{tabular}{cccccc}
\hline \multirow{2}{*}{ Censoring rate } & Parameters & \multicolumn{2}{c}{ UP } & \multicolumn{2}{c}{ IP } \\
\cline { 2 - 5 } & & Lower limit & Upper limit & Lower limit & Upper limit \\
\hline$\alpha_{1}=2.5$ & 0.1283 & 2.6787 & 0.1639 & 2.9583 \\
$\alpha_{2}=3.0$ & 0.1342 & 3.3523 & 0.1701 & 3.9277 \\
$\beta_{1}=1.6$ & 0.2666 & 3.3633 & 0.3523 & 3.0044 \\
$\beta_{2}=1.3$ & 0.2618 & 3.2837 & 0.3397 & 3.1605 \\
$\lambda_{1}=0.3$ & 0.1991 & 0.4885 & 0.1733 & 0.4310 \\
$\lambda_{2}=0.4$ & 0.1996 & 0.4907 & 0.3733 & 0.5910 \\
$p_{1}=0.5$ & 0.1368 & 2.2408 & 0.1763 & 1.9733 \\
& & & & \\
& $\alpha_{1}=2.5$ & 0.1386 & 2.8999 & 0.1722 & 3.1662 \\
$\alpha_{2}=3.0$ & 0.1362 & 3.6547 & 0.1745 & 4.1778 \\
$\beta_{1}=1.6$ & 0.2964 & 3.4257 & 0.3631 & 3.1373 \\
$\beta_{2}=1.3$ & 0.2712 & 3.7151 & 0.3592 & 3.5716 \\
$\lambda_{1}=0.3$ & 0.2034 & 0.4903 & 0.2764 & 0.4933 \\
$\lambda_{2}=0.4$ & 0.2065 & 0.4990 & 0.3746 & 0.5920 \\
$p_{1}=0.5$ & 0.1452 & 3.1401 & 0.1805 & 2.4102 \\
\hline
\end{tabular}




\section{Biographies}

Rahila Yousaf obtained her MPhil from Quaidi-Azam University, Islamabad, and is currently a $\mathrm{PhD}$ statistics candidate in the Department of Mathematics and Statistics at Riphah International University, Islamabad. Her research interests include Bayesian inference, and mixture distributions.

Sajid Ali obtained a PhD in Statistics from Bocconi University, Milan, Italy, and is currently Assistant Pro- fessor in the Department of Statistics at Quaid-i-Azam University (QAU), Islamabad, Pakistan. His research interests include Bayesian inference, construction of new flexible probability distributions, and process monitoring.

Muhammad Aslam obtained his $\mathrm{PhD}$ degree in Statistics from the University of Wales, UK and is currently Professor of Statistics at Riphah International University, Islamabad, Pakistan. His research interests include Bayesian inference and paired comparison models. 